\title{
Vehicle State and Tyre Force Estimation: Demonstrations and Guidelines
}

\author{
Marco Viehweger ${ }^{\mathrm{a}}$, Cyrano Vaseur ${ }^{\mathrm{b}}$, Sebastiaan van Aalst ${ }^{\mathrm{b}}$, Manuel Acosta ${ }^{\mathrm{c}}$, Enrico \\ Regolin $^{\mathrm{d}}$, Angel Alatorre ${ }^{\mathrm{e}}$, Wim Desmet ${ }^{\mathrm{a}}$, Frank Naets ${ }^{\mathrm{a}}$, Valentin Ivanov ${ }^{\mathrm{c}}$, Antonella \\ Ferrara $^{\mathrm{d}}$, and Alessandro Victorino ${ }^{\mathrm{e}}$ \\ ${ }^{a}$ Department of Mechanical Engineering, KU Leuven, Belgium and Flanders Make DMMS-M \\ @ KU Leuven; ${ }^{\mathrm{b}}$ Flanders Make DecisionS, Belgium and Department of Mechanical \\ Engineering, KU Leuven; 'Fakultät für Maschinenbau, TU Ilmenau, Germany; \\ ${ }^{\mathrm{d}}$ Dipartimento di Ingegneria Industriale e dell'Informazione, University of Pavia, Italy; \\ eUniversité de Technologie de Compiègne, France
}

\author{
ARTICLE HISTORY \\ Compiled December 23, 2019
}

\begin{abstract}
This paper presents an in-depth analysis of the application of different techniques for vehicle state and tyre force estimation using the same experimental data and vehicle models, except for the tyre models. Four schemes are demonstrated: (i) an Extended Kalman Filter (EKF) scheme using a linear tyre model with stochastically adapted cornering stiffness, (ii) an EKF scheme using a Neural Network (NN) datadriven linear tyre model, (iii) a tyre model-less Suboptimal-Second Order Sliding Mode (S-SOSM) scheme, and (iv) a Kinematic Model (KM) scheme integrated in an EKF. The estimation accuracy of each method is discussed. Moreover, guidelines for each method provide potential users with valuable insight into key properties and points of attention.
\end{abstract}

\section{KEYWORDS}

Virtual sensing; State estimation; Sliding Mode Observer; Neural Network; Kalman Filtering; Quaternion; Sideslip angle; COG velocities; Tyre forces.

\section{Introduction}

Advanced Driver Assistance Systems (ADAS) as well as Automated Driving (AD) technologies are being increasingly implemented in mass market vehicles, aiming for improved driving safety and passenger comfort [1]. Those technologies can be enhanced by the knowledge of tyre forces and vehicle planar motion states (longitudinal and lateral velocities of the Centre of Gravity (COG) and sideslip angles).

The sideslip angle in the vehicle's COG is defined as the angle between the vehicle's longitudinal axis and its direction of travel and can be determined from the planar states, being COG velocities and yaw rate [2,3]. Planar tyre forces - and their controlled distribution over the wheels - govern safe motion of the vehicle. Therefore, knowledge of these planar states and tyre forces is crucial for the development and im-

\footnotetext{
CONTACT Marco Viehweger. Email: marco.viehweger@kuleuven.be; Cyrano Vaseur. Email: cyrano.vaseur@flandersmake.be
} 
plementation of ADAS such as Lane Centering Assist (LCA), Autonomous Emergency Braking (AEB), and obstacle avoidance. Vertical tyre forces are essential to enhance passenger comfort through (semi-) active suspension control.

This work deals with techniques estimating tyre forces and planar motion states based on readily available measurement data and models. Those virtual sensors have clear benefits over their physical counterparts such as being far less intrusive and expensive. Theoretically, the planar vehicle velocities can be measured using GPS. However, low cost sensors show poor accuracy and robustness which can only be improved with a more expensive GPS unit. Both options are undesired. Similarly, tyre forces can, for example, be measured by Wheel Force Transducers (WFTs) [4], which are extremely costly though. In contrast, virtual sensors for the planar vehicle velocities and the tyre forces require only non-intrusive sensors: Inertial Measurement Unit (IMU), GPS, wheel speed sensors and steering angle sensor.

However, some effort needs to be spend on modelling the vehicle's behaviour as a foundation for the estimators. In this work we distinguish between two model categories, denoting them as kinematic when no forces and inertia are present for the estimation, and dynamic when we do consider these effects explicitly.

Kinematic models are used for state estimation [5]. [6] discusses a kinematic approach for sideslip angle estimation. The main advantage of these models is robustness against changes in vehicle tyre and road parameters. In contrast, in the absence of dynamics and consequently state updating, estimations heavily depend on measurements as model inputs. Therefore, the sensitivity for sensor noise and bias is high. But also for measurement corruption, for example the negative effect of body and road angles on IMU data.

Dynamic models allow the estimation of both planar velocities and tyre forces. They often use a range of vehicle dynamics sub-models. For example, the longitudinal tyre forces can be estimated through a rotating wheel dynamics model, the lateral (peraxle) tyre forces through a bicycle model, and the vertical tyre forces through a load transfer model.

Further distinction is made based on the integrated tyre model. Nonlinear tyre models, such as the Brush, Dugoff, LuGre, or Magic Formula models, are well known [7-14]. Numerous works employing such models for tyre force estimation can be found [15-18]. Stable estimations are obtained using these deterministic models. The drawback here is the risk of estimation bias when using incorrect tyre and road parameters. This is not unlikely to occur due to the high number of varying parameters in such models, for example tyre inflation pressure, effective rolling radius, and road condition (tyre/road friction coefficient). Alternatively, an adaptive tyre model can be used [19], for example, the adaptive linear tyre model [20]. To capture nonlinear behaviour, linear model parameters can be estimated stochastically [21] or using sliding modes. Another option are data-driven techniques, for example Neural Networks (NN) [22-25]. Finally, tyre model-less estimation schemes can be applied [26-29]. Here, direct tyre force estimation can be executed, using for example Sliding Mode Observers [30,31].

Many vehicle state and tyre force estimation techniques have been proposed and partially compared [32]. These include Kalman Filters [33,34], Neural Networks [22,35,36], observers, both Luenberger [37] and Sliding Modes [38,39]. This paper demonstrates four model-based schemes for vehicle state and tyre force estimation. Three of the methods use the same basic vehicle dynamics sub-models (excluding tyre models). Hence, similar accuracy levels are expected for the different methods. Therefore, the focus is rather on some key guidelines per method, required to achieve the presented performance. With this in mind, it is more interesting to have more rather than less 
diversity amongst the methods. Therefore, several distinctions are made, leading to the following choices: (i) an Extended Kalman Filter (EKF) scheme using a linear tyre model with stochastically adapted cornering stiffness, (ii) an EKF scheme using a Neural Network (NN) data-driven linear tyre model and (iii) a tyre model-less Suboptimal-Second Order Sliding Mode (S-SOSM) scheme. (iv) Finally, these dynamic model-based methods are distinguished from a kinematic model-based approach (KM) which is integrated in an EKF. The same reference data is used for demonstrating their accuracy performance. Furthermore, guidelines are discussed for each of the methods. These list the key points for the development of the techniques in order to obtain the presented accuracy performance.

The rest of the paper is structured in the following manner: Section 2 explains the vehicle model which is the base for the estimation procedures. An introduction to the electric test vehicle, the test track, and the recorded data is given in Section 3. The employed estimation approaches are presented in Section 4. Section 5 discusses the estimation results in comparison to the reference signals and provides general points of attention as well as guidelines for successful implementation. Finally, Section 6 delivers the conclusions and points for future work.

\section{Vehicle modelling}

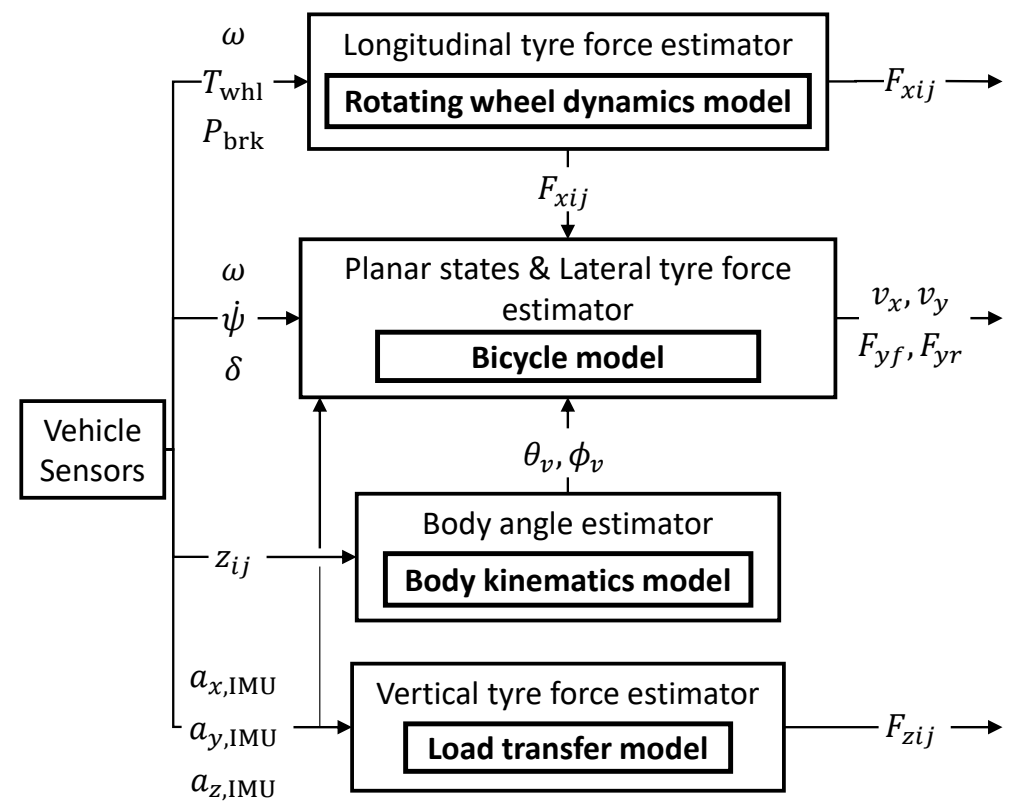

Figure 1. Sub-models used in general estimation framework.

This chapter treats the different sub-models used in the general estimation framework (see Fig. 1) of the methods demonstrated in this paper. As already mentioned in the introduction, rotating wheel dynamics are employed for longitudinal tyre force estimation. A bicycle model is used in the estimators to obtain the planar states and the planar tyre forces (except for the KM approach). A body kinematics model is used in combination with suspension position $\left(z_{i j}\right)$ sensors to calculate body angles required to correct IMU data when used in the planar states estimators. A quasi-static load 
transfer model is applied to calculate the vertical tyre forces from measured accelerations.

For this paper, only forward driving has been considered as most significant dynamics (especially in lateral direction) are expected in this case. However, the employed estimation schemes are anticipated to also work for backward driving.

\subsection{Planar vehicle dynamics}

The estimation techniques compared in this paper are based on the bicycle model, as proposed by Segel [40]. The model is shown in Fig. 2, where $\delta$ is the steering angle, $\dot{\psi}$ the yaw rate, $v_{x}$ and $v_{y}$ are the centre of gravity (COG) vehicle speeds, $\beta, \alpha_{f}$ and $\alpha_{r}$ the COG, front and rear axle sideslip angles, $F_{x f}$ and $F_{x r}$ the front and rear axle longitudinal tyre forces, $F_{y f}$ and $F_{y r}$ the front and rear axle lateral tyre forces, and $l_{f}$ and $l_{r}$ are the distances from the COG to the front and rear axle, respectively.

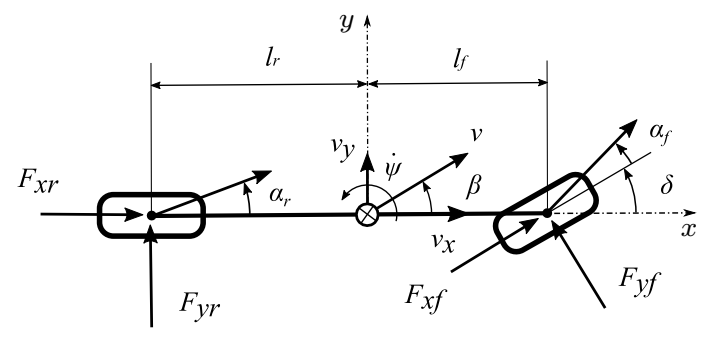

Figure 2. The bicycle model, as developed by [40].

With this model the planar vehicle dynamics can be formulated as:

$$
\begin{aligned}
m\left(\dot{v}_{x}-\dot{\psi} v_{y}\right) & =F_{x f} \cos (\delta)-F_{y f} \sin (\delta)+F_{x r}-F_{\text {res }} \\
m\left(\dot{v}_{y}+\dot{\psi} v_{x}\right) & =F_{y f} \cos (\delta)+F_{x f} \sin (\delta)+F_{y r} \\
I_{z z} \ddot{\psi} & =l_{f}\left(F_{y f} \cos (\delta)+F_{x f} \sin (\delta)\right)-l_{r} F_{y r}
\end{aligned}
$$

in which $m$ is the vehicle mass and $I_{z z}$ the yaw moment of inertia. The influence of air and rolling resistance on the longitudinal force equilibrium is described by a second-order polynomial:

$$
F_{\text {res }}=C_{0}+C_{1} v_{x}+C_{2} v_{x}^{2}
$$

in which the coefficients $C_{k}$ are defined to match the test vehicle:

$$
C=\left[\begin{array}{l}
297 \mathrm{~N} \\
8.34 \mathrm{~N} \mathrm{~s} / \mathrm{m} \\
0.49 \mathrm{~N} \mathrm{~s}^{2} / \mathrm{m}^{2}
\end{array}\right]
$$




\subsection{Rotating wheel dynamics}

The longitudinal tyre forces $F_{x i j}$ are described according to the rotating wheel dynamics:

$$
\left\{\begin{aligned}
I_{\mathrm{whl} l} \dot{\omega}_{i j} & =T_{\mathrm{drv} i j}-T_{\mathrm{brk} i j}-r_{l}\left(F_{x i j}+\mu_{r} F_{z i j}\right) \\
T_{\mathrm{brk} i j} & =k_{\mathrm{brk}} P_{\mathrm{brk} i j}
\end{aligned}\right.
$$

Here and throughout the rest of this paper $i j$ is used to denote the four vehicle corners: $i \in\{\boldsymbol{f}$ ront, $\boldsymbol{r e a r}\}, j \in\{\boldsymbol{l}$ eft, $\boldsymbol{r i g h t}\} . I_{\text {whl }}$ and $\omega_{i j}$ are the wheel rotational inertia and speed. $T_{\mathrm{drv} i j}$ and $T_{\mathrm{brk} i j}$ are the driving and braking torques. The braking torque is calculated from the hydraulic brake pressure $P_{\text {brkij }}$ through the proportional constant $k_{\text {brk. }}$. Finally, $r_{l}$ is the loaded tyre radius and $\mu_{r}$ is the rolling resistance coefficient.

\subsection{Load transfer}

The quasi-static load transfer model (5) is used to describe the normal tyre forces $F_{z i j}$, cf. $[14,41]$. Road angles are neglected. Roll and pitch motion are assumed decoupled. The model captures vehicle weight $m g$ which is distributed over the front and rear axle according to the position of the COG $\left(l_{f}, l_{r}\right)$. The effect of vehicle pitch (due to longitudinal acceleration $a_{x}$ ) on the normal forces is also accounted for. In both cases load on the axle is distributed over left and right according to the COG position $\left(t_{l}, t_{r}\right)$. Finally, the effect of vehicle roll is taken into account. Hereby the loads are distributed over the front and rear axle according to $k_{\phi f}$ and $k_{\phi r}$, the front and rear equivalent roll stiffnesses taking into account suspension springs as well as anti-roll bars. A tuning procedure based on (5) comparing computed and measured wheel load $F_{z i j}$ for the rear axle revealed the $k_{\phi i}$ values; the roll centre height $h_{\phi i}$ was deduced from the suspension geometry. With a symmetric suspension, the load change on the left and right tyres due to vehicle roll is equal and opposite.

$$
\left\{\begin{aligned}
\Delta F_{z \phi i}= & \frac{1}{T}\left(\frac{k_{\phi i}}{k_{\phi f}+k_{\phi r}-m a_{z \mathrm{IMU}} h^{\prime}} h^{\prime}+\frac{L-l_{i}}{L} h_{\phi i}\right) \\
& m a_{y \mathrm{IMU}} \\
\Delta F_{z \theta i}= & \pm \frac{m a_{x \mathrm{IMU}} h}{2 L} \\
F_{z i j}= & \frac{T-t_{j}}{T} \cdot \frac{\left(L-l_{i}\right) m a_{z \mathrm{IMU}}}{L} \pm \Delta F_{z \phi i} \pm \Delta F_{z \theta i}
\end{aligned}\right.
$$

Here, $h^{\prime}$ is the perpendicular distance between the COG and the roll axis. The geometric dimensions and force quantities are shown in Fig. 3.

\subsection{Vehicle body kinematics}

Vehicle body roll and pitch angles $\phi_{v}, \theta_{v}$ are calculated kinematically from four suspension stroke sensors measuring $z_{i j}$. The approach is adopted from [42]. Four estimates are made per body roll and pitch angle. Each estimate is based on a combination of three suspension sensors. Finally, per body angle, the average is taken over the four estimates. 


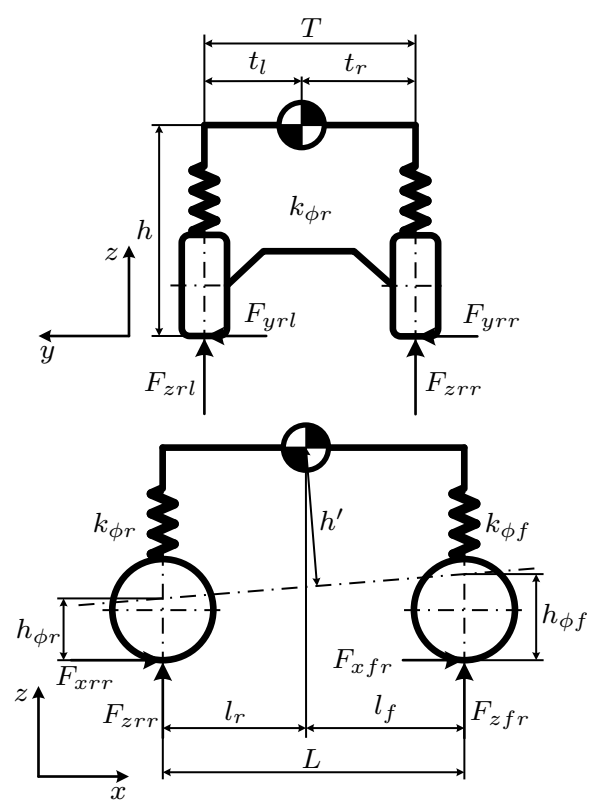

Figure 3. Tyre forces and vehicle geometry.

For the estimation of the planar states and tyre forces, the IMU acceleration measurements $a_{x \mathrm{IMU}}, a_{y} \mathrm{IMU}$ need to be corrected from errors introduced by gravity $g(6)$. Therefore, the vehicle body angles as shown in Fig. 4 are required.

$$
\left\{\begin{array}{l}
a_{x \mathrm{IMU} \text { corr }}=a_{x \mathrm{IMU}}+g \sin \left(\theta_{v}\right) \\
a_{y \mathrm{IMU} \text { corr }}=a_{y} \mathrm{IMU}-g \sin \left(\phi_{v}\right)
\end{array}\right.
$$

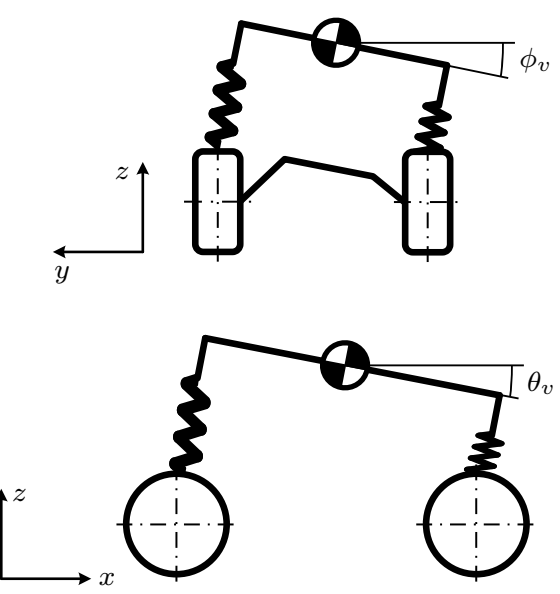

Figure 4. Vehicle body angles.

In this paper, road bank and slope angles are neglected as the track on which the test data was recorded is quite flat and thus the influence of the road angles on the estimation accuracy is expected to be very limited. However, approaches for road angles estimation can be found in literature, for example [42].

The IMU measurements corrected from vehicle body angle influences still need to be 


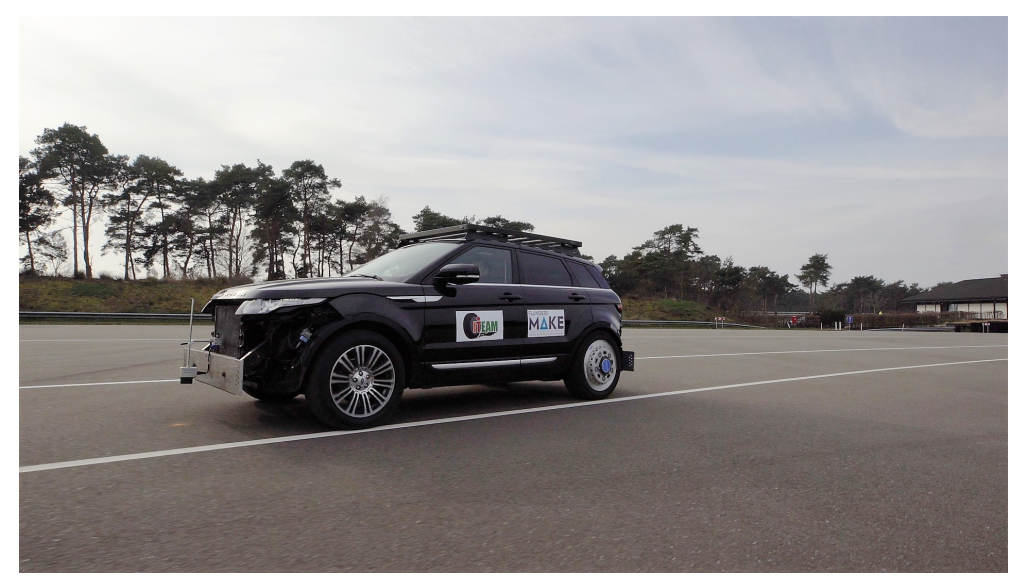

Figure 5. Test vehicle: Electrified SUV.

transformed from the position of the IMU to the COG. [43] gives a clear explanation of this process based on the following equation, where $\mathbf{a}$ is the vector of the COG accelerations:

$$
\mathbf{a}=\mathbf{a}_{\mathrm{IMU} \operatorname{corr}}-\dot{\varphi} \times\left(\dot{\varphi} \times \mathbf{r}_{\mathrm{IMU}}\right)-\ddot{\varphi} \times \mathbf{r}_{\mathrm{IMU}}
$$

Here, $\dot{\varphi}$ is the vector of the vehicle body's angular velocities, $\ddot{\varphi}$ is the vector of the vehicle body's angular accelerations, and $\mathbf{r}_{\mathrm{IMU}}$ is the vector of the IMU to COG distances as specified in Table 1. Please note that the IMU is installed in the horizontal plane of the COG such that only longitudinal and lateral distances occur.

\section{Test vehicle and data}

\subsection{Vehicle}

The vehicle dynamics tests have been conducted with Flanders Make's electrified compact SUV, see Fig. 5. Table 1 contains the specifications of the fully equipped vehicle, including driver and passenger in the front seats. It features two independent electric motors at the rear axle; however, torque vectoring control had been disabled for the data acquisition (nevertheless, the employed estimation schemes are anticipated to deliver valid estimated signals also in the case of active torque vectoring control). Two high-fidelity WFTs were installed in the rear.

In order to record the required estimator input, measurement, and reference signals, the following sensors have been used: WFTs measuring the three-dimensional rear wheel forces, optical sensor for vehicle body velocity and sideslip angle reference, IMU to capture the translational accelerations and angular velocities of the vehicle body, suspension stroke sensors for body angles calculation. Additional signals have been retrieved via the vehicle's CAN bus: steering wheel angle (with known mapping to angle of left and right front wheels), angular wheel speeds, and brake pressure. The electric motors' torque signals were acquired from the motor controllers. The data rate of all sensor signals has been limited to $100 \mathrm{~Hz}$ as this was the lowest physical data rate amongst the installed sensors.

A calibration procedure was performed before each data collection test ensuring 
Table 1. Test vehicle specifications.

\begin{tabular}{|c|c|c|c|}
\hline & & Value & Unit \\
\hline \multicolumn{4}{|l|}{ Body geometry } \\
\hline Wheelbase & $L$ & 2.675 & $\mathrm{~m}$ \\
\hline Distance between front axle and COG & $l_{f}$ & 1.439 & $\mathrm{~m}$ \\
\hline Distance between rear axle and COG & $l_{r}$ & 1.236 & $\mathrm{~m}$ \\
\hline Track width & $T$ & 1.625 & $\mathrm{~m}$ \\
\hline Distance between left track and COG & $t_{l}$ & 0.778 & $\mathrm{~m}$ \\
\hline Distance between right track and $\mathrm{COG}$ & $t_{r}$ & 0.847 & $\mathrm{~m}$ \\
\hline Height of COG above ground & $h$ & 0.65 & $\mathrm{~m}$ \\
\hline Vehicle mass & $m$ & 2,442 & $\mathrm{~kg}$ \\
\hline Yaw inertia & $I_{z z}$ & 3,231 & $\mathrm{~kg} \mathrm{~m}^{2}$ \\
\hline Longitudinal distance between IMU and COG & $r_{\mathrm{IMU} x}$ & 0.580 & $\mathrm{~m}$ \\
\hline Lateral distance between IMU and COG & $r_{\mathrm{IMU} y}$ & 0.070 & $\mathrm{~m}$ \\
\hline \multicolumn{4}{|l|}{ Suspension, wheel, brake system } \\
\hline Front equivalent roll stiffness & $k_{\phi f}$ & 95,300 & $\mathrm{Nm} / \mathrm{rad}$ \\
\hline Rear equivalent roll stiffness & $k_{\phi r}$ & 123,400 & $\mathrm{Nm} / \mathrm{rad}$ \\
\hline Front roll centre height & $h_{\phi f}$ & 0.19 & $\mathrm{~m}$ \\
\hline Rear roll centre height & $h_{\phi r}$ & 0.07 & $\mathrm{~m}$ \\
\hline Loaded tyre radius & $r_{l}$ & 0.343 & $\mathrm{~m}$ \\
\hline Rolling resistance coefficient & $\mu_{r}$ & 0.01 & \\
\hline Wheel rotational inertia & $I_{\mathrm{whl}}$ & 0.9 & $\mathrm{~kg} \mathrm{~m}^{2}$ \\
\hline Front proportional constant & $k_{\mathrm{brk} f}$ & 36.3 & $\mathrm{Nm} / \mathrm{bar}$ \\
\hline Rear proportional constant & $k_{\mathrm{brk} r}$ & 9.2 & $\mathrm{Nm} / \mathrm{bar}$ \\
\hline \multicolumn{4}{|l|}{ Powertrain } \\
\hline Electric motor power, nominal & & $2 \times 42$ & $\mathrm{~kW}$ \\
\hline Electric motor torque, nominal & & $2 \times 135$ & $\mathrm{Nm}$ \\
\hline
\end{tabular}

that signals recorded by the WFTs were correctly adjusted to zero or their steadystate value.

\subsection{Experimental test data}

All tests have been carried out with the electrified SUV at the Ford Lommel Proving Ground (LPG) in Belgium.

Two laps on the vehicle handling track, aka. inner durability track or 'Track 7', of the LPG were performed: first one with mild vehicle dynamics excitation, resembling everyday driving conditions, followed by a limit handling lap with very high excitation levels. Fig. 6 provides an overview of the track; the star marks start and finish of the laps, the blue dot indicates a cobblestone corner, and the red triangles highlight a hilly road section. Fig. 7 and 8 show the vehicle behaviour during the low and high excitation laps, respectively.

The figures showing the estimation results in Section 5 are based on the fast Track 7 lap. For reference however, error metric tables for both slow and fast lap are included.

To complement the two laps on Track 7 , tests with different standard vehicle handling manoeuvres were performed: Steady-state constant radius, ISO double lane change, slalom, step steer, straight line braking, braking in turn. 


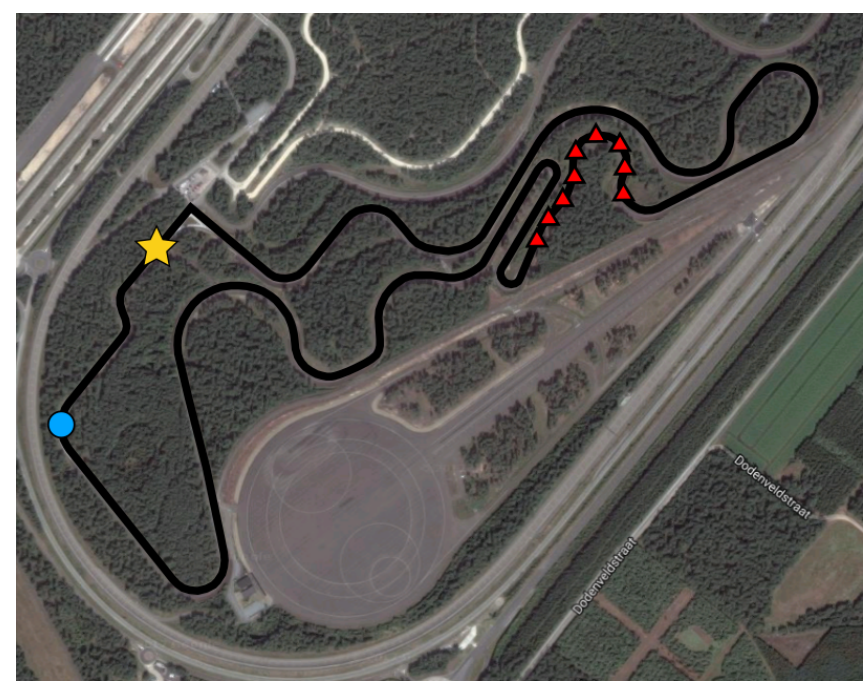

Figure 6. Test track: 'Track 7' at Ford LPG.
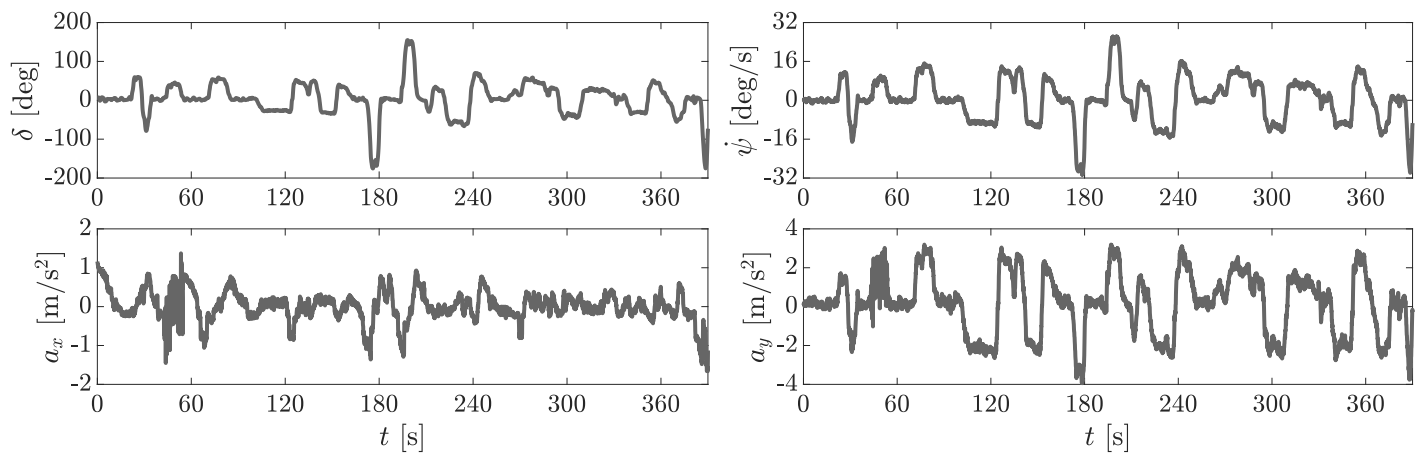

Figure 7. Measurements for slow Track 7 lap.

\section{Estimation approaches}

This paper compares a total of four vehicle state and tyre force estimation approaches:

- Purely Linear and Extended Kalman Filter based ('KF');

- Neural Network and Extended Kalman Filter based ('NN');

- Suboptimal-Second Order Sliding Mode and Extended Kalman Filter based ('SSOSM');

- Kinematic model and Extended Kalman Filter based velocity estimation ('KM').

The four approaches have been selected to compare rather traditional with more recent techniques: KF only requires the well-established EKF; NN employs Neural Networks for tyre modelling; S-SOSM is based on a recently proposed enhancement of Sliding Mode Control; KM makes use of quaternion notation for ground vehicle state estimation which has not been demonstrated before.

Two approaches (KF and NN) provide estimates of the planar vehicle states (longitudinal, lateral velocities $v_{x}, v_{y}$, and yaw rate $\dot{\psi}$ ) as well as longitudinal and lateral (per-axle) tyre forces $F_{x}, F_{y}$; the S-SOSM approach is solely used to estimate $F_{x}$ and $F_{y}$; with KM only the planar vehicle states are estimated, cf. Table 2. A common 

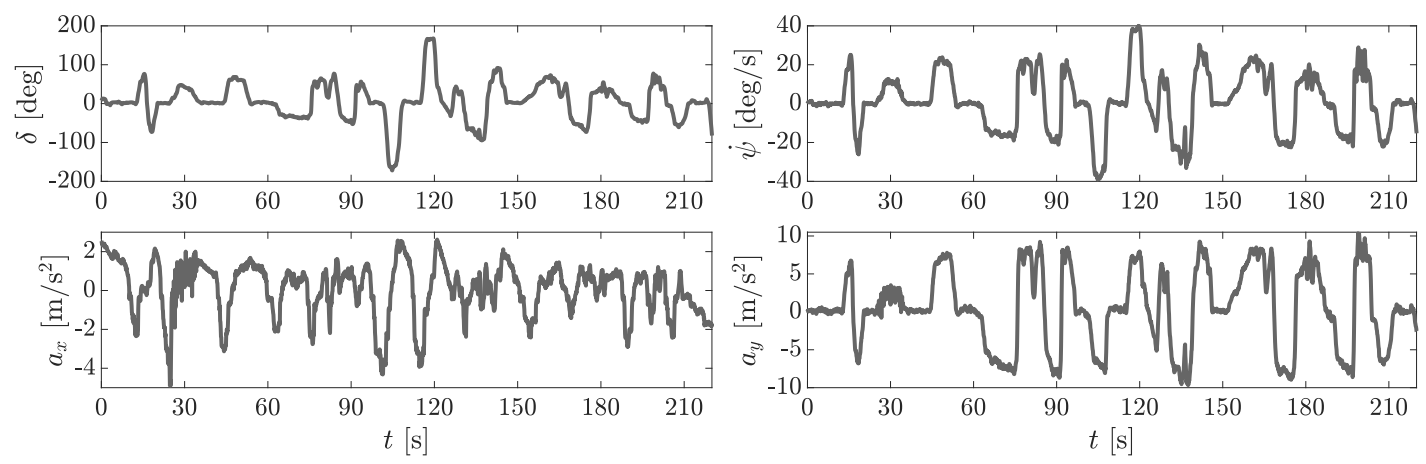

Figure 8. Measurements for fast Track 7 lap.

Table 2. Estimation approach overview.

\begin{tabular}{lcc}
\hline Approach & State est. & Tyre force est. \\
\hline KF & $\checkmark$ & $\checkmark$ \\
NN & $\checkmark$ & $\checkmark$ \\
S-SOSM & & $\checkmark$ \\
KM & $\checkmark$ & \\
\hline
\end{tabular}

approach is employed to obtain the vertical tyre forces $F_{z}$.

\subsection{Vertical tyre forces}

In this paper the common approach for obtaining the vertical tyre forces $F_{z i j}$ is based on the load transfer model (5):

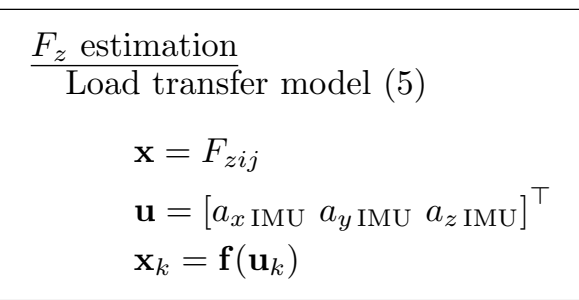

\subsection{Linear and Extended Kalman Filter approach}

The KF approach is comprised of two estimator modules. In the first module, the individual longitudinal tyre forces are estimated. A discrete-time KF is constructed based on the wheel rotational dynamics (4), augmented with a random walk model for the longitudinal tyre forces. The time-discretisation is performed by a forward Euler scheme. The estimated longitudinal tyre forces are taken as input to the second estimator module, in which the axle lateral tyre forces and vehicle planar motion states are estimated. This module uses a discrete-time EKF built on the bicycle model (1).

The second estimator module has two specific features that require further explanation. Firstly, the dynamic state update equation for the longitudinal velocity (1a) is replaced by a kinematic one:

$$
\dot{v}_{x}=a_{x}+\dot{\psi} v_{y}
$$




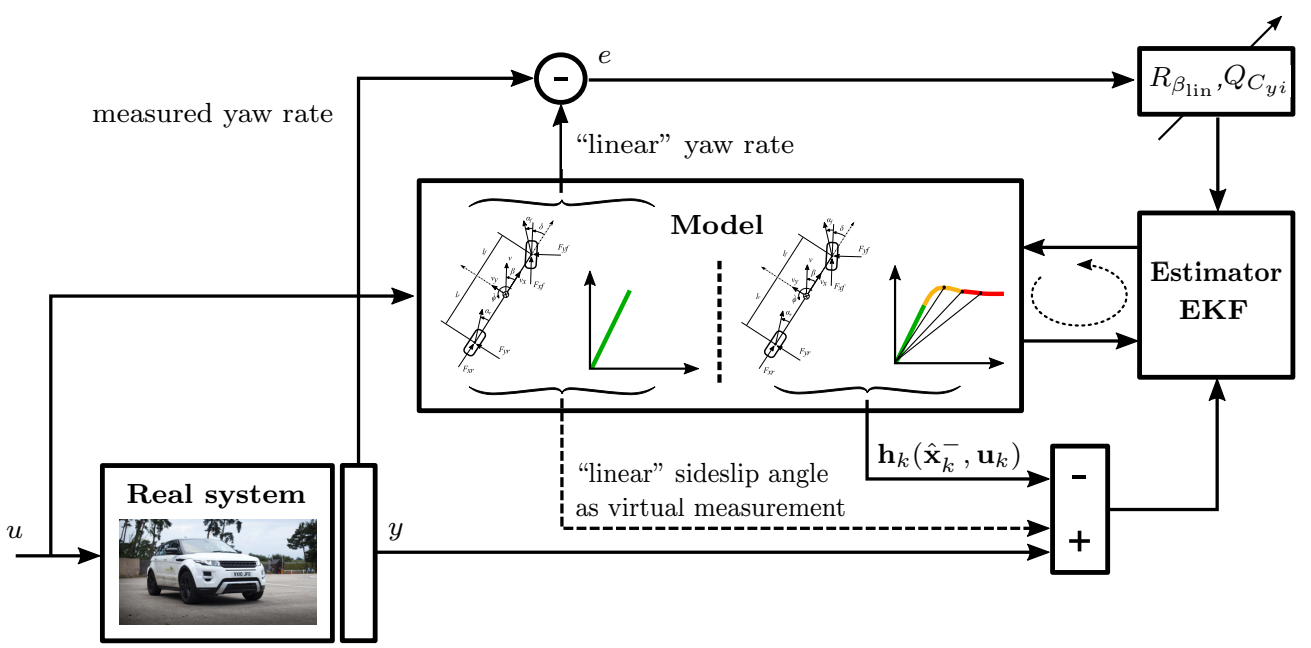

Figure 9. Scheme of the axle lateral tyre force and vehicle planar motion state estimator of the KF-based approach.

with $a_{x}$ the longitudinal acceleration according to (7). The predictions from this model are corrected by the wheel speed measurements, whereby a best wheel selection algorithm is employed that selects the measurement from the wheel with the least amount of longitudinal slip. This 'best' wheel speed measurement is denoted as $v_{x \text {,best }}$. Secondly, the axle lateral tyre forces are modelled by the adaptive linear tyre model proposed in [20]:

$$
\left\{\begin{array}{l}
F_{y i}=-2 C_{y i} \alpha_{i} \\
\dot{C}_{y i}=0
\end{array} \text { for } i \in\{f, r\}\right.
$$

where $\alpha_{i}$ is the axle sideslip angle and $C_{y i}$ is the unknown axle cornering stiffness, whose evolution is represented by a zero-order hold model with a predefined uncertainty in the Kalman Filter to account for variable tyre behaviour (nonlinear tyre behaviour, road friction changes, etc.). The axle sideslip angles are given by the following kinematic relations (under a small angle assumption):

$$
\alpha_{f}=\frac{v_{y}+l_{f} \dot{\psi}}{v_{x}}-\delta, \quad \alpha_{r}=\frac{v_{y}-l_{r} \dot{\psi}}{v_{x}}
$$

As was shown in previous work of the authors [21,34], the resulting system exhibits 'excitation dependent' observability. That is, its observability becomes gradually poorer as the axle sideslip angles tend to zero, eventually leading to an unobservable system for straight driving. This leads to poor estimation performance in low excitation situations (situations in which the sideslip angles remain small). To overcome this problem, an enhanced estimation scheme was proposed in [21]. This scheme exploits the fact that linear tyre behaviour is mostly a property of the tyre and to a much lesser extent of the road conditions. As a consequence, in case of linear tyre behaviour, the sideslip angle may be accurately predicted by a vehicle model that assumes linear tyre behaviour, irrespective of the road conditions. The proposed scheme makes such prediction, denoted $\beta_{\text {lin }}$, and adds it as a pseudo measurement in the estimator. In case of nonlinear tyre behaviour, the estimator relies on the adaptive tyre model and applies cornering stiffness adaptation to track this nonlinear behaviour. In the same 
way robustness to varying road conditions is obtained. A covariance adaptation strategy is then applied to assign appropriate uncertainties to this pseudo measurement and to the unknown cornering stiffnesses: the more nonlinear the tyres behave, the higher the uncertainties assigned to $\beta_{\operatorname{lin}}$ and $C_{y i}$. To this end, a degree of nonlinearity measure in tyre behaviour is defined based on a continuous yaw rate criterion. This is further explained in the guidelines, Section 5.2.

Fig. 9 schematically illustrates this approach, which successfully mitigates the issues related to poor observability in low excitation situations while maintaining proper performance in high excitation situations and robustness to varying road conditions. The complete estimation process in step-by-step overview:

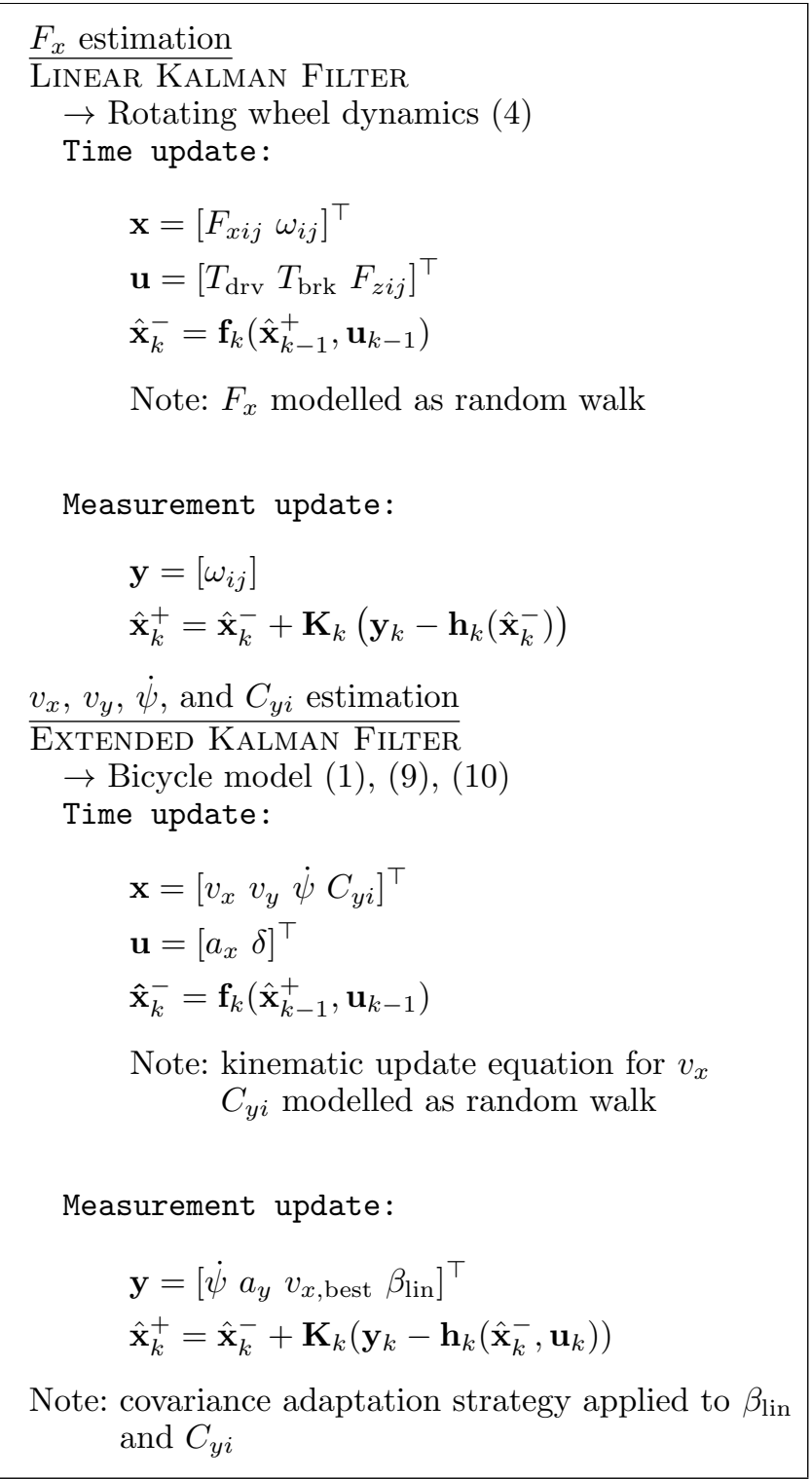

\subsection{Neural Network and Extended Kalman Filter approach}

Similar to the KF estimation approach and following the methodology introduced in previous works $[24,29,44]$, different state estimation structures are constructed and 
integrated in a modular fashion as displayed in Fig. 1 and Fig. 10. This section is primarily focused on the tyre cornering stiffness estimation method, being the main difference from the KF approach.

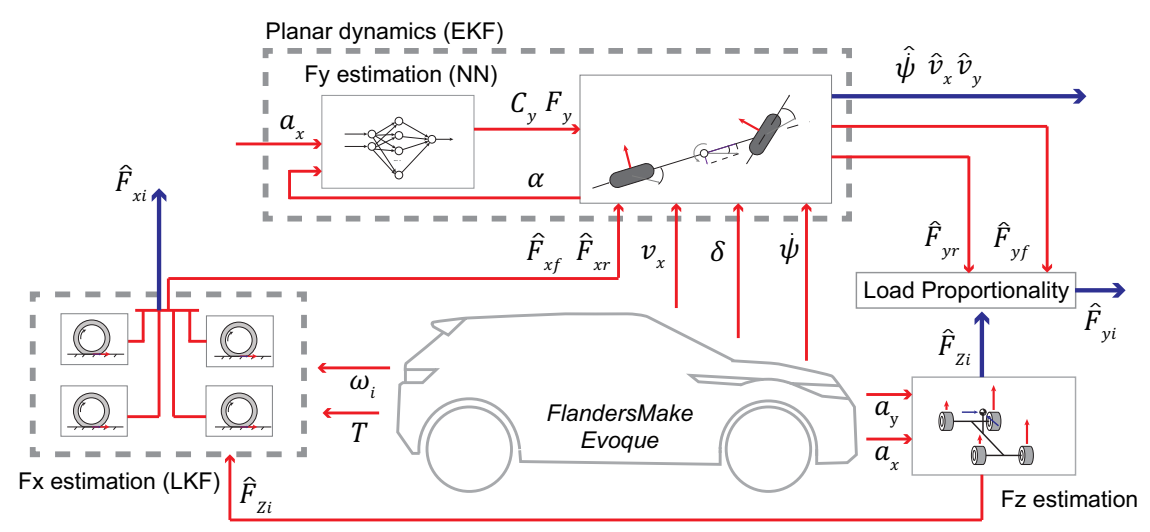

Figure 10. Modular NN-based virtual sensor.

Equivalent to the KF method, a tyre model of linear characteristic is employed here. The axle lateral forces are modelled as:

$$
F_{y i} \approx F_{y i 0}+\Delta F_{y i}=F_{y i 0}+C_{y i} \Delta \alpha_{i}
$$

Specifically, the axle lateral force $F_{y i}$ is defined as the sum of a steady-state term $F_{y i 0}$ and a force increment $\Delta F_{y i}$. The latter term is formed by the product of the axle lateral stiffness $C_{y i}$ and the axle lateral slip increment $\Delta \alpha_{i}$. The terms $F_{y i 0}$ and $C_{y i}$ are considered time-varying EKF parameters, and are approximated by a feedforward NN structure.

This approach is aimed at avoiding the conventional tyre characterisation procedure (i.e. sandpaper tyre testing, skid-trailer tyre testing [45-47]) by means of an in-vehicle standardised tyre testing programme. This methodology was first introduced and validated through a software-in-the-loop (SIL) programme in [24] for testing the NN's robustness to tyres of different size and operating at different conditions (e.g. tyre pressure, time-varying friction).

The NNs are trained to fit the nonlinear functions $F_{y f}=f_{\mathrm{NN} f}\left(a_{x}, \alpha_{f}\right), F_{y r}=$ $f_{\mathrm{NN} r}\left(a_{x}, \alpha_{r}\right)$, where the longitudinal acceleration was introduced to capture the axle lateral force reduction experienced during combined-slip situations [24]. In order to determine these functions, the objective testing manoeuvres presented in Table 3 were executed with Flanders Make's electrified SUV at Ford LPG.

Table 3. Objective testing manoeuvres to train the axle lateral force NNs.

\begin{tabular}{lr}
\hline Manoeuvre & Repetitions \\
\hline Braking in a turn & 4 \\
Steady-state constant radius & 14 \\
Step steer & 5 \\
\hline
\end{tabular}

These manoeuvres are standardised steady-state and transient tests often performed to characterise relevant chassis attributes such as the yaw responsiveness, yaw damping 
or understeering gradient [48]. The number of repetitions was dictated by the data available after performing the experimental activities described in Section 3.2. For simplicity, all the repetitions were considered as an initial step. As accurate results were obtained with this reduced number of manoeuvres it was not necessary to perform additional iterations over the training dataset.

The NN training datasets were formed by direct concatenation of each test run. In particular, an input dataset was formed by the longitudinal acceleration signals acquired from a high-accuracy IMU and the axle lateral slip computed from (10). The yaw rate and longitudinal velocity signals were logged directly from the vehicle CAN bus, while the lateral velocity was obtained from an optical sensor mounted at the front bumper (this signal was translated to the vehicle COG for consistency). The output datasets were formed by the axle lateral forces experienced by the chassis. In this case, the rear axle lateral force was defined as the sum of the lateral forces measured by the WFTs, $F_{y r}=F_{y r l}+F_{y r r}$, while the front axle lateral force was reconstructed offline adopting a synthesised planar dynamics model.

Once the training datasets were defined, two NN structures were created adopting the net. $m$ Matlab function and trained using a Levenberg-Marquardt backpropagation algorithm. This step was realised by means of the trainlm.m function. The number of hidden-layer neurons was set to 6 (i.e. 2-6-1 NN structures) following a systematic design methodology and the NN stability was studied following the approach detailed in [24]. After approximating the nonlinear functions $f_{\mathrm{NN} f}, f_{\mathrm{NN} r}$, the axle lateral stiffness values $C_{y i}$ were computed following a finite differences approach. For additional details regarding the previous steps [24] can be consulted.

From a vehicle implementation point of view, the proposed structure can be easily embedded in current Yaw Stability Control (YSC) systems. Specifically, an accurate estimate of the lateral velocity is provided from a standard set of on-board measurements. The additional tyre friction information estimated by the structure can be employed to infer the road friction potential following a slip-based estimation approach [24].

\subsection{Suboptimal-Second Order Sliding Mode and Extended Kalman Filter approach}

This approach consists of two stages: the first one is a tyre force observer relying on the so-called Suboptimal-Second Order Sliding Mode (S-SOSM) [49]; the second stage is an EKF enhancement allowing to smoothen the S-SOSM estimates alongside the sensor signals.

\subsubsection{S-SOSM-based observer}

Fig. 11 shows this part of the estimation approach schematically. The three main elements are (i) longitudinal tyre force observer, (ii) wheel torque adaptation, and (iii) lateral tyre force observer.

4.4.1.1. Longitudinal tyre force observer. The longitudinal tyre force $F_{x i j}$ estimation employs the rotating wheel dynamics (4) in a slightly altered form (see (12)) and is performed in the wheel frame. Measured wheel speeds $\omega_{i j}$ and requested wheel torques $T_{i j}=T_{\mathrm{drv} i j}-T_{\mathrm{brk} i j}$ are used to generate an S-SOSM on the sliding surface 


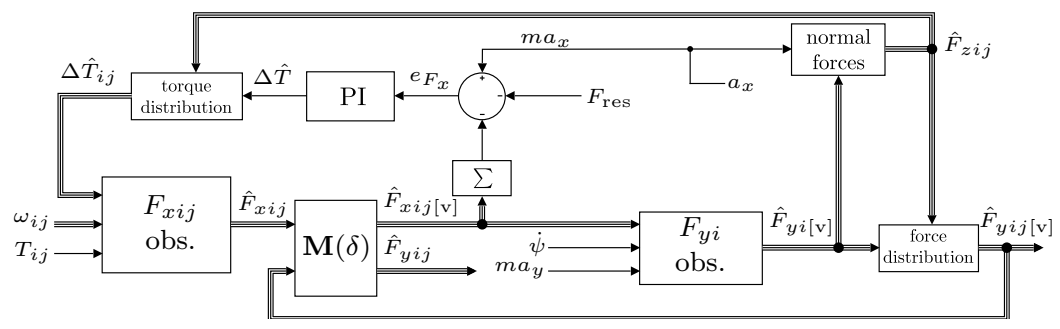

Figure 11. The S-SOSM observation scheme.

$\sigma_{x i j}=\omega_{i j}-\hat{\omega}_{i j}$. This task is performed with the estimation law

$$
I_{\text {whl }} \dot{\hat{\omega}}_{i j}=T_{i j}-\Delta \hat{T}_{i j}-r_{l} u_{x i j}
$$

where $u_{x}$ is the observer input law [49] defined by

$$
u_{x / y}=-\int K_{x / y} \operatorname{sign}\left(\sigma_{x / y}-\frac{\sigma_{x / y \max }}{2}\right) \mathrm{d} t
$$

with gain $K_{x / y}$ for the longitudinal and lateral case respectively and $\sigma_{x / y \max }=$ $\sigma_{x / y}\left(t_{\max }\right)$, where $t_{\max }$ is the last time instant at which $\dot{\sigma}_{x / y}=0$. The estimated longitudinal tyre force is $\hat{F}_{x w}=u_{x}$.

4.4.1.2. Wheel torque adaptation. The overall torque deviation $\Delta \hat{T}$ in (12) is the output of the PI adaptive law:

$$
\Delta \dot{\hat{T}}=K_{P} \dot{e}_{F_{x}}+K_{I} e_{F_{x}}
$$

Based on (1), the input $e_{F_{x}}$ is given by:

$$
e_{F_{x}}=m a_{x}-\left(\sum_{i j}\left(\hat{F}_{x i j[\mathrm{v}]}\right)-F_{\text {res }}\right)
$$

with $F_{\text {res }}$ computed according to (2).

The term $\Delta \hat{T}$ is then allocated on the individual wheels $\left(\Delta \hat{T}_{i j}\right.$ in (12)) taking the driving situation into account. It is assumed that, when a negligible torque is applied to a specific wheel, the corresponding torque deviation is null. In case of a rear wheel driven vehicle, such as the one considered in the experimental validation in Section 3.2, the following $\Delta \hat{T}$ distribution is applied:

$$
\Delta \hat{T}_{i j}=\left\{\begin{array}{lll}
\Delta \hat{T} \frac{\hat{F}_{z r j}}{\hat{F}_{z r}} & \text { if } a_{x}>a_{\mathrm{thd}} & \text { acceleration } \\
\Delta \hat{T} \frac{\hat{F}_{z i j}}{m g} & \text { if }\left|a_{x}\right|<a_{\mathrm{thd}} & \text { coasting } \\
\Delta \hat{T} \frac{\hat{F}_{z i j}}{m g} c_{\mathrm{brk}} & \text { if } a_{x}<-a_{\mathrm{thd}} & \text { braking }
\end{array}\right.
$$

with the normal forces estimated according to (5), the coefficient $c_{\text {brk }}$ accounting for the brake torque split ratio, and $a_{\text {thd }}$ being a calibratable threshold.

4.4.1.3. Lateral tyre force observer. A single S-SOSM observer is sufficient for the estimation of the lateral tyre forces based on the single track vehicle model (1) and 
the relation $m a_{y}=F_{y f[\mathrm{v}]}+F_{y r[\mathrm{v}]}$, with the lateral forces being expressed in the vehicle body frame. In order to account for differential tyre force induced yaw moments, the term $\Delta \hat{F}_{x i[\mathrm{v}]}(i \in\{f, r\})$ is added to (1c) [31]. The following S-SOSM observer is designed to steer the estimation error $\sigma_{y}=\dot{\psi}-\dot{\hat{\psi}}$ to zero in finite time, with $\ddot{\hat{\psi}}$ and $\hat{F}_{y f[\mathrm{v}]}$ defined as

$$
\left\{\begin{array}{l}
\ddot{\hat{\psi}}=\frac{1}{I_{z z}}\left[l_{f} m a_{y}-\left(l_{f}+l_{r}\right) u_{y}+\frac{T}{2} \Delta \hat{F}_{x f[\mathrm{v}]}+\frac{T}{2} \Delta \hat{F}_{x r[\mathrm{v}]}\right] \\
\hat{F}_{y f[\mathrm{v}]}=m a_{y}-u_{y}
\end{array}\right.
$$

As in the longitudinal case, the estimated force acting on the rear axle is $\hat{F}_{y r[\mathrm{v}]}=u_{y}$, where $u_{y}$ is again the output of the S-SOSM control law (13). Note that, the forces summation in (15) is expressed in the body frame, so that a linear transformation is required to convert the estimated values $\hat{F}_{x i j}$ provided by (12). This computation is implemented by means of a rotation matrix $\mathbf{M}(\delta)$, for which more details are provided in $[31]$.

\subsubsection{EKF enhancement}

The adoption of the EKF allows for smoothing the estimated tyre/axle forces $F_{x i j}$, $F_{y i}$, as well as the acquired measurement signals $\dot{\psi}, a_{y}$, and $a_{x}$ used to obtain them.

The EKF, see for example [33], is implemented with the state, measurement, and input vectors $\mathbf{x}, \mathbf{y}$, and $\mathbf{u}$ being defined as:

$$
\begin{aligned}
& \mathbf{x}=\left[\begin{array}{llllll}
F_{x i j} & F_{y r[\mathrm{v}]} & a_{x} & a_{y} & \omega_{i j} & \dot{\psi}
\end{array}\right]^{\top} \\
& \mathbf{y}=\left[\hat{F}_{x i j}^{\mathrm{SM}} \hat{F}_{y r[\mathrm{v}]}^{\mathrm{SM}} a_{x}^{\text {sens }} a_{y}^{\text {sens }} \omega_{i j}^{\text {sens }} \dot{\psi}^{\text {sens }}\right]^{\top} \\
& \mathbf{u}=\left[T_{i j} \delta\right]^{\top}
\end{aligned}
$$

and superscripts sens and SM denoting 'sensor measurement' and 'sliding mode estimation' respectively. Moreover, since we directly measure the state, $\mathbf{H}(t)=\mathbf{H}=\mathbf{I}$ holds. In order to apply the EKF, we assume known process noise $\mathbf{w} \sim \mathcal{N}(0, \mathbf{Q})$ and measurement noise $\mathbf{v} \sim \mathcal{N}(0, \mathbf{R})$, in the nonlinear continuous state-space description

$$
\dot{\mathbf{x}}=\mathbf{f}(\mathbf{x}, \mathbf{u}, \mathbf{w}), \quad \mathbf{y}=\mathbf{h}(\mathbf{x}, \mathbf{v})
$$

The nonlinear function $\mathbf{f}(\mathbf{x}, \mathbf{u}, \mathbf{w})$ has the form

$$
\mathbf{f}(\mathbf{x}, \mathbf{u}, \mathbf{w})=\left[0,0,0,0,0,0,0, f_{x i j}, f_{y}\right]
$$

where the terms $f_{x}, f_{y}$, which in practice are obtained from (1) and (4), depend on vehicle states and parameters. Their explicit definition can be found in [31].

The EKF covariance matrices $\mathbf{Q}$ and $\mathbf{R}$ were tuned by running an optimisation procedure. Non-diagonal elements were set to zero in order to reduce the computational cost. The results of this optimisation for the values of diagonal elements of $\mathbf{Q}$ and $\mathbf{R}$ as well as the observer gains $\mathbf{K}$ are reported in Table 4 . As is evident by the number of parameters, the calibration effort for the S-SOSM method is considerable. However, this is mainly for the EKF enhancement. In fact, thanks to this smoothing stage, there is no need to reduce the gains $\mathbf{K}$ for chattering alleviation purposes, as for example in [50]. The output of the S-SOSM observer can be seen as a noisy measurement for the EKF, thus requiring a proper tuning of the observation covariance matrix $\mathbf{R}$. 
Table 4. S-SOSM tuning parameters.

\begin{tabular}{ccccccc}
\hline$Q_{F_{x}}$ & $Q_{F_{y}}$ & $Q_{a_{x}}$ & $Q_{a_{y}}$ & $Q_{\omega}$ & $Q_{\dot{\psi}}$ & $K_{x}$ \\
26.03 & 7.68 & 70.35 & 8.93 & 93.28 & 77.86 & $2 \cdot 10^{6}$ \\
\hline$R_{F_{x}}$ & $R_{F_{y}}$ & $R_{a_{x}}$ & $R_{a_{y}}$ & $R_{\omega}$ & $R_{\dot{\psi}}$ & $K_{y}$ \\
0.25 & 0.03 & 0.1 & 0.05 & 0.02 & 2 & $2 \cdot 10^{3}$ \\
\hline
\end{tabular}

\subsection{Kinematic model and Extended Kalman Filter approach}

In order to estimate the planar vehicle states, this approach employs the following kinematic model:

$$
\begin{gathered}
\mathbf{v}=v_{x} \boldsymbol{i}+v_{y} \boldsymbol{j}+v_{z} \boldsymbol{k} \\
\mathbf{a}=\dot{\mathbf{v}}+\dot{\boldsymbol{\varphi}} \times \mathbf{v}
\end{gathered}
$$

Here, $\mathbf{v} \in \mathbb{R}^{3}$ and $\mathbf{a} \in \mathbb{R}^{3}$ represents the velocity and acceleration vectors respectively. Furthermore, $\dot{\mathbf{v}} \in \mathbb{R}^{3}$ and $\dot{\varphi} \in \mathbb{R}^{3}$ denote the velocity time derivative and the angular rates respectively. Model equation (19) is extended to include the effects of gravity and the quaternion definition is used to model rotations, leading to:

$$
\left[\begin{array}{l}
0 \\
\mathbf{a}
\end{array}\right]=\left[\begin{array}{c}
0 \\
\dot{\mathbf{v}}
\end{array}\right]+\left[\begin{array}{c}
0 \\
\dot{\boldsymbol{\varphi}} \times \mathbf{v}
\end{array}\right]+\mathbf{q} \otimes\left[\begin{array}{l}
0 \\
\mathbf{g}
\end{array}\right] \otimes \mathbf{q}^{*}
$$

Here, $\mathbf{g} \in \mathbb{R}^{3}$ describes the gravity vector, $\mathbf{q}, \mathbf{q}^{*} \in \mathbb{H}^{4}$ represent a quaternion and its conjugate respectively. Finally $\otimes$ stands for the Hamiltonian product.

The complete system is defined as:

$$
\begin{aligned}
{\left[\begin{array}{c}
0 \\
\dot{\mathbf{v}}
\end{array}\right] } & =\left[\begin{array}{c}
0 \\
\mathbf{a}
\end{array}\right]-\left[\begin{array}{c}
0 \\
\dot{\boldsymbol{\varphi}} \times \mathbf{v}
\end{array}\right]-\mathbf{q} \otimes\left[\begin{array}{l}
0 \\
\mathrm{~g}
\end{array}\right] \otimes \mathbf{q}^{*} \\
\dot{\mathbf{q}} & =\frac{1}{2} \mathbf{q} \otimes \dot{\boldsymbol{\varphi}}
\end{aligned}
$$

System (21) is nonlinear given the cross product and the Hamiltonian product. Finally, based on (21) an EKF is built to estimate the longitudinal and lateral vehicle speeds.

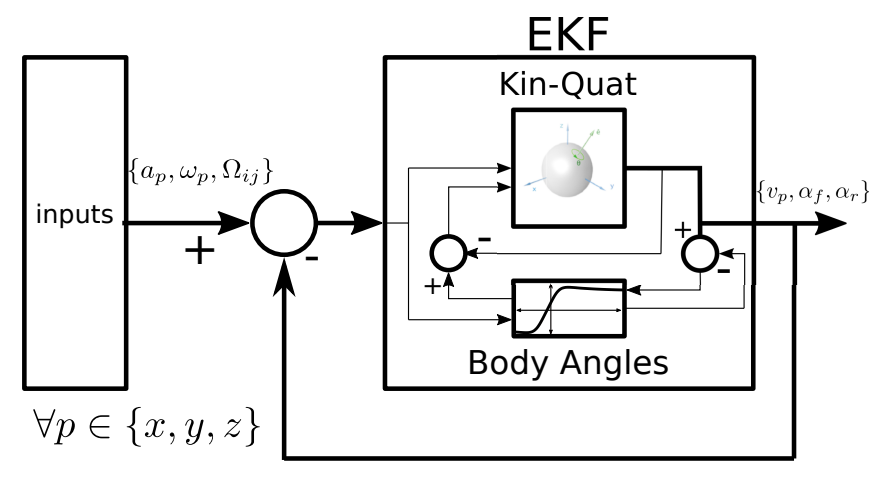

Figure 12. Lateral and longitudinal speed observation scheme.

Figure 12 shows the observer scheme. The inputs required are given by an inertial measurements unit, $a_{p}$ and $\dot{\varphi}_{p}, \forall p \in\{x, y, z\}$, and the wheel speed sensors, $\omega_{i j}$. Again, 
the suffix $i j$ refers to a specific vehicle corner: $i \in\{\boldsymbol{f}$ ront, $\boldsymbol{r e a r}\}, j \in\{\boldsymbol{l}$ eft, $\boldsymbol{r i g h t}\}$.

The observer estimates the longitudinal and lateral velocities with respect to the position of the accelerometers and gyroscopes. Thus, it is imperative to know these parameters in advance. If they are unknown, the performance of the algorithm can still be improved via the tuning of the EKF.

Tuning of the estimation approach can be done by 'trial-and-error', see [5], the random walk variable related covariances are with a higher order compared to the ones measured. In this case an optimisation technique was implemented to define the covariance matrices $\mathbf{Q}$ and $\mathbf{R}$. Note that the tuning optimisation process is based on different data sets than the ones presented in the results section of this paper (Section 5).

\section{Discussion of results and guidelines}

The following Fig. 13 to Fig. 17 show the estimation results of all methods in direct comparison with the reference signals, where applicable; as the test vehicle was equipped with WFTs only at the rear axle, no front tyre force reference signals were recorded. However, those signals can be estimated, of course. For brevity, the figures only show the data of the fast Track 7 lap.

In order to counteract the noise level exhibited by the optical reference velocity sensor, a third order, zero-phase digital Butterworth filter with $5 \mathrm{~Hz}$ cutoff frequency has been applied during data post-processing on the plotted longitudinal and lateral velocity reference signals. This ensures the preservation of the signals' relevant frequency content and still reduces the noise enough to allow a graphical comparison in Fig. 16 and Fig. 17.

Each observer was tuned individually but then the tuning was kept constant for all results presented in this work. In general, all estimation approaches track the reference signals closely and with comparable accuracy. For an objective comparison of the estimation results against the reference, Root-Mean-Square Error (RMSE) and maximum error $\left(e_{\max }\right)$ are computed as follows:

$$
\begin{aligned}
& R M S E=\sqrt{\frac{\left\|x-x_{\mathrm{ref}}\right\|^{2}}{N_{s}}} \\
& e_{\max }=\max \left(\left|x-x_{\mathrm{ref}}\right|\right)
\end{aligned}
$$

where $x$ is the estimated quantity, $x_{r e f}$ the reference signal, and $N_{s}$ the number of samples in the complete time series.

Tables 5 and 6 contain the results. Fig. 18 displays the normalised RMSE and normalised $e_{\max }$ error metrics for both slow and fast Track 7 laps. Smaller normalised error values imply higher estimation accuracy and thus better results.

The dynamic model-based KF and NN approaches perform sufficiently accurate when it comes to the estimation of the planar states, assuming a planar vehicle model. The dynamic model-based characteristic of the estimators leads to clean estimates with low levels of noise. Similar accuracy in slow lap (rely mostly on linear model), and fast lap (rely mostly on adaptive linear model), demonstrate the proper functioning of the $\mathrm{KF}$ approach for tyre force virtual sensing. For the NN approach, a limited number of field tests is sufficient to capture the tyre characteristics and supply accurate and 

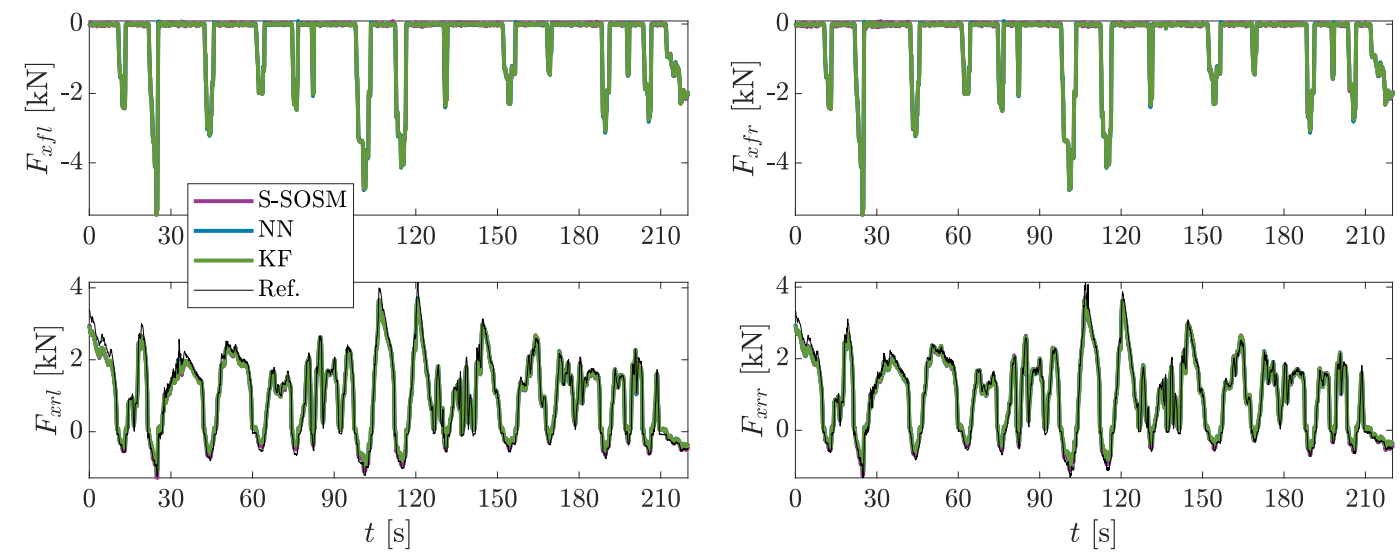

Figure 13. Longitudinal tyre forces for fast Track 7 lap.

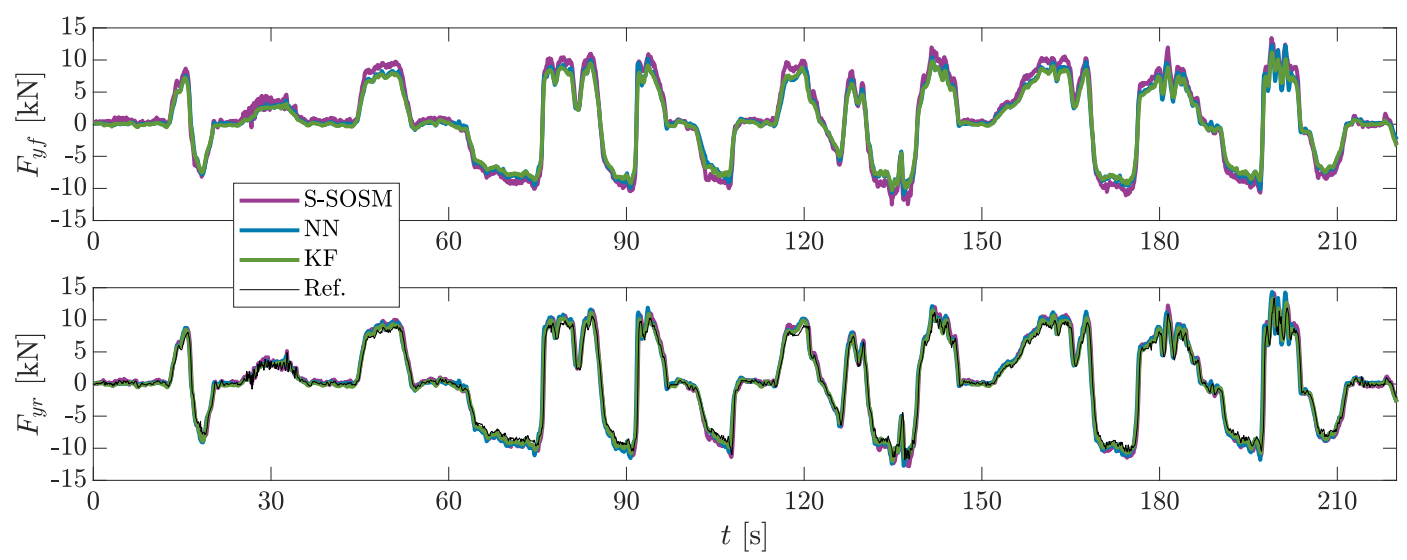

Figure 14. Lateral axle tyre forces for fast Track 7 lap.

low-noise tyre force estimates.

Concerning the S-SOSM approach, tyre force estimation accuracy is similar to the other approaches - with a slightly higher effort: by using the enhanced vehicle model (16) within the estimation algorithm and assuming correct vehicle parameters, one 'forces' the estimation to be correct in (finite) short time, not considering the chattering. Then the EKF enhancement allows to remove the chattering, at the cost of a minor delay introduced by the filtering.

The KM approach delivers similar results for the planar vehicle states compared to the other methods using dynamic models, despite its simplicity. Generally, the results are noisier - due to the lack of the low-pass filtering effect of the vehicle modelbut tracking accuracy is similar. However, a more involved approach making use of quaternions is needed to handle the IMU orientation correctly.

\subsection{Vertical tyre force estimation - guidelines}

The load transfer model (5) is used for vertical tyre force estimation and is a less integral part of the estimation scheme as opposed to the other vehicle sub-models. Signal exchange is unidirectional. Estimated vertical tyre forces are pre-calculated 

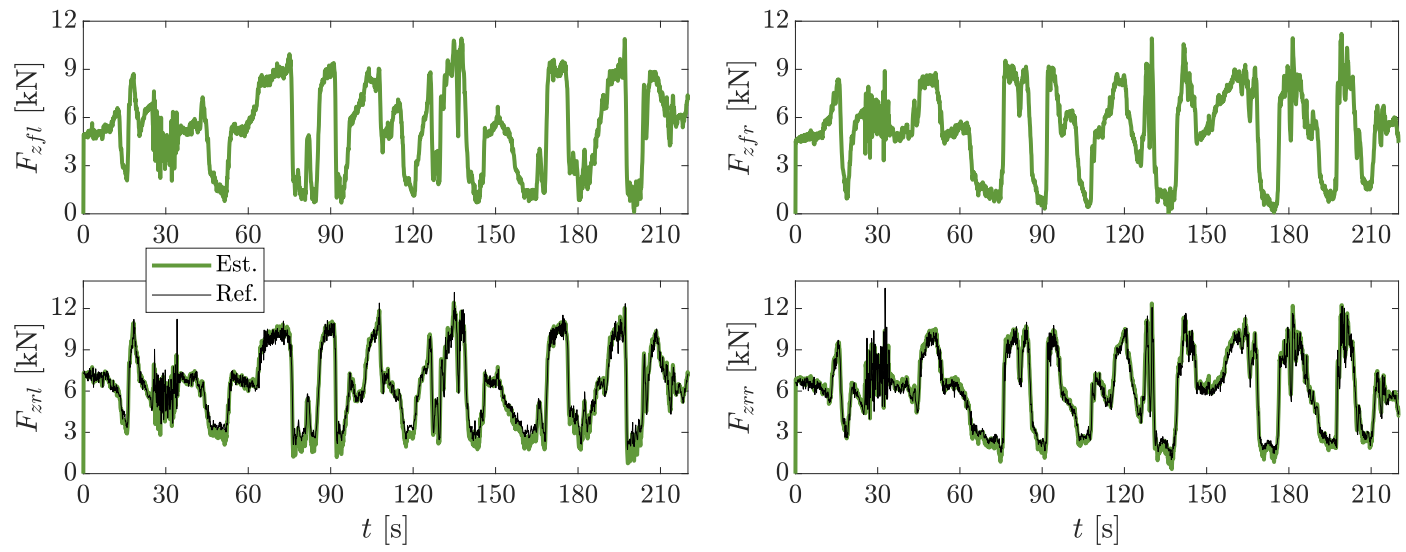

Figure 15. Vertical tyre forces for fast Track 7 lap.

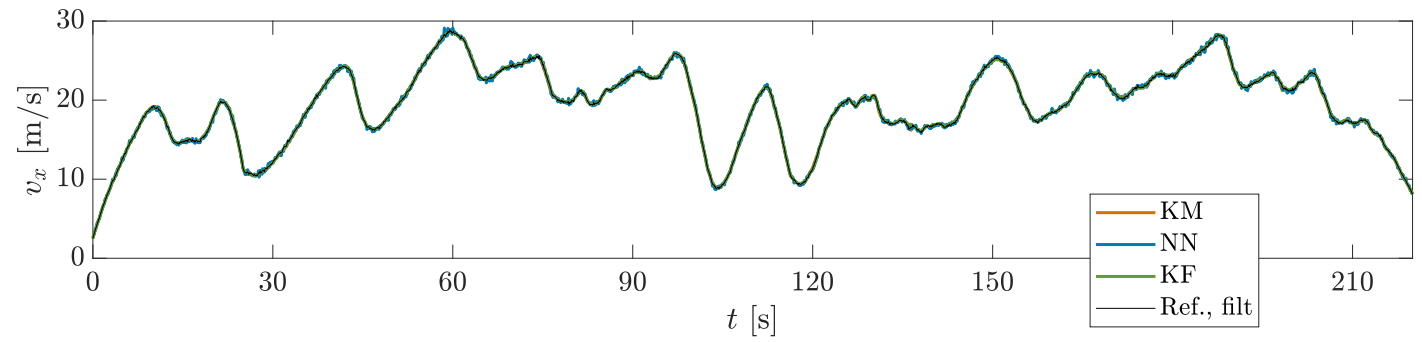

Figure 16. Longitudinal vehicle velocity for fast Track 7 lap.

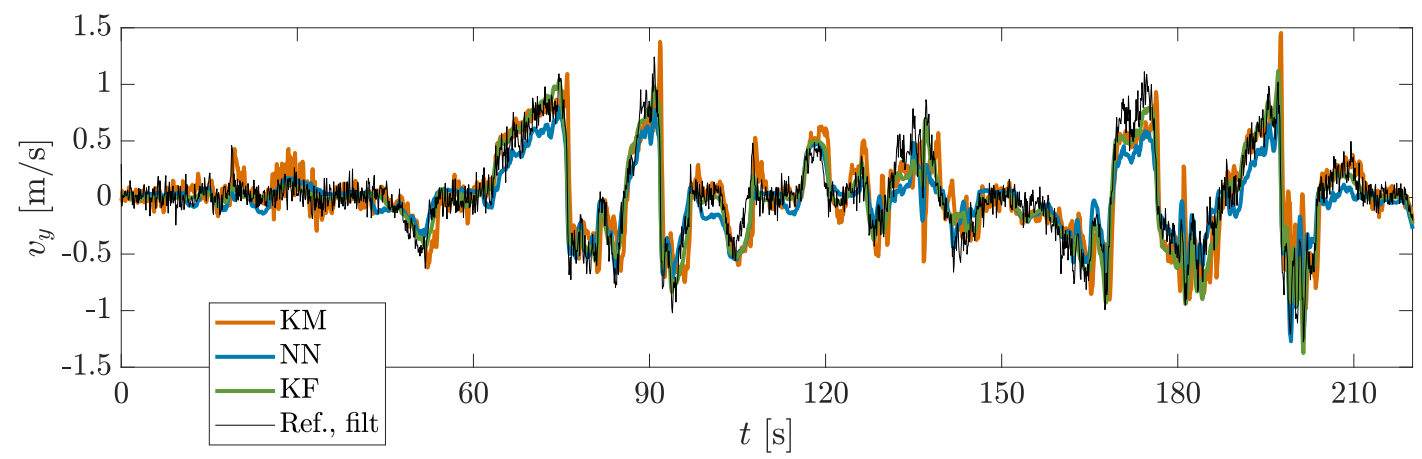

Figure 17. Lateral vehicle velocity for fast Track 7 lap. 
Table 5. Error metrics for slow Track 7 lap

\begin{tabular}{|c|c|c|c|c|c|c|c|}
\hline & \multicolumn{3}{|c|}{ RMSE } & \multicolumn{3}{|c|}{$e_{\max }$} & \multirow[b]{2}{*}{ Unit } \\
\hline & S-SOSM & NN & $\overline{K F}$ & $\overline{\text { S-SOSM }}$ & NN & $\overline{K F}$ & \\
\hline \multirow{4}{*}{$\begin{array}{l}F_{x} \\
F_{y} \\
F_{z} \\
\end{array}$} & 110 & 107 & 109 & 441 & 417 & 446 & $\mathrm{~N}$ \\
\hline & 257 & 411 & 406 & 1936 & 2099 & 2131 & $\mathrm{~N}$ \\
\hline & \multicolumn{3}{|c|}{348} & \multicolumn{3}{|c|}{6906} & $\mathrm{~N}$ \\
\hline & KM & NN & $\mathbf{K F}$ & KM & NN & KF & \\
\hline$v_{x}$ & 0.21 & 0.25 & 0.21 & 0.91 & 1.05 & 0.92 & $\mathrm{~m} / \mathrm{s}$ \\
\hline$v_{y}$ & 0.22 & 0.21 & 0.21 & 1.11 & 0.80 & 0.82 & $\mathrm{~m} / \mathrm{s}$ \\
\hline
\end{tabular}

Table 6. Error metrics for fast Track 7 lap

\begin{tabular}{|c|c|c|c|c|c|c|c|}
\hline & \multicolumn{3}{|c|}{ RMSE } & \multicolumn{3}{|c|}{$e_{\max }$} & \multirow[b]{2}{*}{ Unit } \\
\hline & S-SOSM & NN & $\mathbf{K F}$ & S-SOSM & NN & $\mathbf{K F}$ & \\
\hline \multirow{4}{*}{$\begin{array}{l}F_{x} \\
F_{y} \\
F_{z}\end{array}$} & 184 & 183 & 190 & 1146 & 1095 & 1114 & $\mathrm{~N}$ \\
\hline & 700 & 1358 & 755 & 2744 & 14104 & 6940 & $\mathrm{~N}$ \\
\hline & \multicolumn{3}{|c|}{625} & \multicolumn{3}{|c|}{7258} & $\mathrm{~N}$ \\
\hline & KM & NN & $\mathbf{K F}$ & KM & NN & $\mathbf{K F}$ & \\
\hline$v_{x}$ & 0.28 & 0.33 & 0.28 & 1.44 & 1.44 & 1.36 & $\mathrm{~m} / \mathrm{s}$ \\
\hline$v_{y}$ & 0.36 & 0.31 & 0.29 & 2.05 & 1.46 & 1.27 & $\mathrm{~m} / \mathrm{s}$ \\
\hline
\end{tabular}

and used as inputs for the estimation of the planar tyre forces.

As the calculations are purely deterministic, the accuracy of the results heavily depends on correct model parameters and measurement inputs of the load transfer model. It is critical to use precise vehicle mass and geometry as well as accurate suspension characteristics. Here, model inputs are the measured COG accelerations.

\subsection{Dynamic model-based approaches - guidelines}

\subsubsection{Longitudinal tyre force estimation}

The uncertainties assigned to the wheel speed measurements are based on the actual variances of the signals. These variances are mainly determined by noise. Since the measurement model is the identity matrix, no model error is present in the measurement model so all uncertainty is related to the signal itself.

Assigning the uncertainties on the model equations is mainly based on physical insights. We know that the rotating wheel dynamics model (4) is rather accurate, so the assigned variance should be small. Similarly, the variance assigned to the random walk model of the longitudinal tyre forces $F_{x}$ is based on its expected variation. After assigning initial guesses based on these insights, further tuning is performed by a 'trial-and-error' procedure.

\subsubsection{COG velocity estimation and cornering stiffness estimation}

As described above, two distinctions are made here.

KF approach: This method estimates the tyre cornering stiffness per axle $C_{y i}$ stochastically as a random walk in an EKF using an adaptive linear tyre model. Additionally, the vehicle sideslip angle $\beta_{\text {lin }}$ is predicted - assuming linear tyre behaviourand added as a pseudo measurement in the estimator. To obtain stable and accurate 
Tyre force estimation

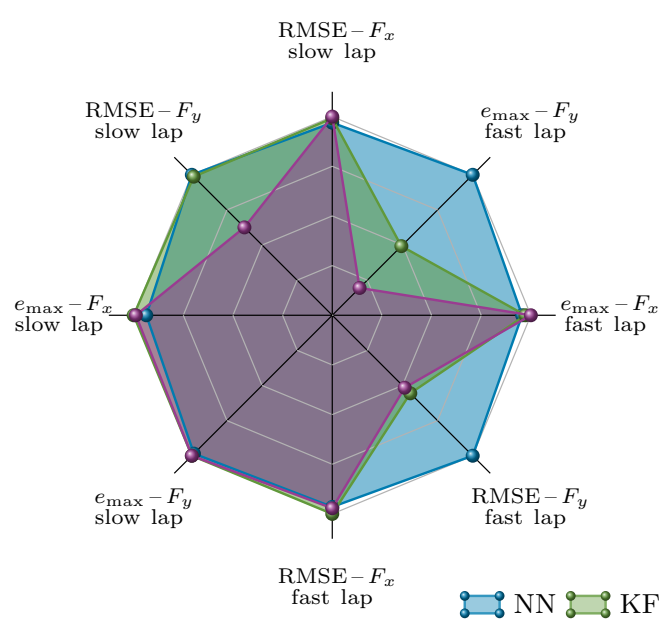

Vehicle state estimation

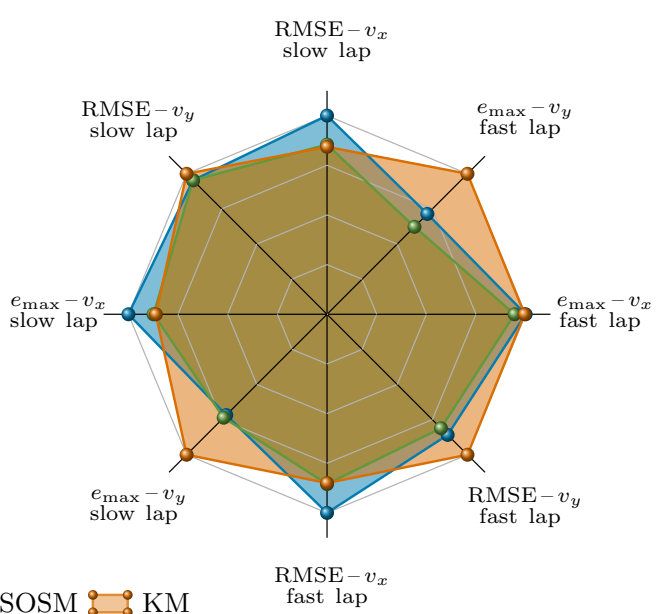

Figure 18. Normalised error metrics for slow and fast Track 7 laps.

performance for low and high driving dynamics, covariance adaptation functions are devised. For nonlinear tyre behaviour, high covariances for $C_{y i}$ and $\beta_{\text {lin }}$ are applied. For linear tyre behaviour, low covariances suffice. Finally, for the case of no lateral excitation, zero value $C_{y i}$ covariance and low $\beta_{\text {lin }}$ covariance are employed. See [21] for further details.

NN approach: In a second method, $C_{y i}$ is estimated using neural networks in a linear tyre model framework. For obtaining good accuracy performance, the NN structure requires proper training. Standardised vehicle dynamics manoeuvres as specified in the estimation approach description, see Section 4.3, are sufficient. This procedure may be easily automated with the development of future automated vehicle testing platforms, cf. [51]. Furthermore, as discussed in Section 4.3, the development of the $\mathrm{NN}$ approach requires reliable data on vehicle velocities and tyre forces. In this study, these are retrieved by an optical velocity sensor and WFTs. Succesfull implementation of this method therefore depends on the presence of such measuring equipment during the development process.

The major concern when dealing with any data-based approach is to guarantee that the training dataset is sufficiently rich so as to avoid potential extrapolation issues. In this sense, large tyre lateral slip angles should be recorded during the execution of the tests (e.g. ramp steer cornering manoeuvres for the front axle or step steer manoeuvres to cause instability on the rear axle). Braking-in-a-turn events should be added to the training dataset in order to capture the tyre force reduction with the longitudinal force. As an example, braking events may be executed at even deceleration levels $(-2$, $-4,-6 \mathrm{~m} / \mathrm{s}^{2}$ ) during steady-state cornering for this purpose.

\subsection{S-SOSM approach-guidelines}

\subsubsection{Longitudinal tyre force estimation}

As opposed to the dynamic model-based approaches, the observer does not update the system states directly, but through the system inputs/observer outputs. In this case, 
the motor torques are the observer inputs. The observer aims to estimate individual longitudinal tyre forces using the rotating wheel dynamics model and the individual wheel speeds. The S-SOSM reference $F_{x}$ is calculated from measured longitudinal acceleration $a_{x}$ and vehicle mass $m$. For this method it is therefore more critical to use an accurate vehicle mass. Also, with high dependency on $a_{x}$, it is crucial to abide by the flat road assumption, or otherwise correct measurements for the road angles induced gravity component. Next to vehicle mass, the accuracy of the wheel radius $r_{l}$ plays a similarly important role in coming to a representative longitudinal force observer reference.

The observer gains have to be chosen based on the dynamics which have to be dominated by control laws (13), as explained extensively in [31].

The EKF smoothing stage of this estimation approach mainly requires tuning effort on the covariance matrices $\mathbf{Q}$ and $\mathbf{R}$. However, in this work an optimisation scheme was used for this task. The non-diagonal elements were chosen zero in order to reduce the computational cost.

\subsubsection{Lateral tyre force estimation}

In contrast to the other methods, direct per-axle tyre force estimation is applied here. No additional effort is required for cornering stiffness estimation. Again, an optimisation scheme was employed for the tuning of the covariance matrices $\mathbf{Q}$ and $\mathbf{R}$.

\subsection{Kinematic model-based approach - guidelines}

\subsubsection{COG velocities estimation}

The greatest benefit of state estimation based on kinematic models is their robustness against parameter variations (e.g. tyre and road parameters but also vehicle mass and inertia parameters) and unknown disturbances. The proposed method use a combination of low cost sensors, and the specific characteristics of these transducers will have a major effect on the performance of the state estimator.

Regularly, kinematic model-based approaches for lateral speed estimation suffer from drift due to observability problems during straight driving. This problem has been solved in [52] and [53] by applying a heuristic function to the planar kinematic model. In our case, System (21) becomes fully observable under random walk considerations even during straight driving.

This method relies on IMU measurements. The angular rates are used in quaternion notation to correct the acceleration bias due to gravity components induced by the body and road angle dynamics. Furthermore, if the geometrical location of the IMU with respect to the desired position of the longitudinal and lateral speed estimation is known beforehand, the uncertainty on the kinematic model is kept at the minimum. These parameters are considered design parameters, therefore they should be known in advance. If they are not, the observation scheme should be tuned accordingly with proper covariance matrices $\mathbf{Q}$ and $\mathbf{R}$. Also time integration errors do not pose issues for this approach, since it is a closed-loop observer and the measurements are considered free from bias. 


\section{Conclusion}

This study demonstrated the use of four model-based approaches for vehicle state and tyre force estimation: (i) an Extended Kalman Filter (EKF) scheme using a linear tyre model with stochastically adapted cornering stiffness, (ii) an EKF scheme using a Neural Network (NN) data-driven linear tyre model, (iii) a tyre model-less SuboptimalSecond Order Sliding Mode (S-SOSM) scheme, and (iv) a Kinematic Model (KM) scheme integrated in an EKF.

Data collected with an electrified and instrumented SUV on a vehicle proving ground is used to generate the input and reference data for all estimation methods. Similar levels of estimation accuracy are obtained by all methods. This is expected as all methods are based on the same vehicle models and model parameters, except for the tyre models.

The method depending more on sensor data (KM) rather than on model predictions ( $\mathrm{KF}$ and $\mathrm{NN}$ ), is more robust at the expense of slightly noisier estimation results. Also, in the absence of state updating, additional steps are required to correct for errors in measurement data. Therefore, the KM approach makes use of a quaternion notation to deal with gravity disturbances on the IMU measurements.

The sensitivity to model parameters for the different methods applied, as discussed in the guidelines section, is not straightforward. For example, the S-SOSM method with its wheel torque adaptation algorithm generates a tyre force reference making direct use of vehicle mass and wheel radius parameters, which need to be as accurate as possible.

Also the tuning effort varies from method to method. For example, to achieve stable and accurate performance with the KF approach, covariance adaptation functions were devised when crossing from linear to nonlinear tyre behaviour. In the case of the NN approach, the training procedure is based on reference data which is typically not available from the standard sensor set installed in production vehicles. In order to identify and suggest solutions for such challenges, this paper contains a dedicated section on guidelines per method.

Finally, applications to ADAS, with its real-time requirements, will further differentiate the methods and their growth paths. This is left as one direction for future research on the demonstrated methods.

\subsection{Future steps}

- Development and implementation of accurate and robust method to infer individual tyre lateral forces from the accurately estimated per-axle signals.

- Implement road bank and slope angle estimation to further enhance applicability of proposed virtual sensors.

- Further comparison of tyre force estimates using a revised tyre force compensation procedure.

- Further comparison of state estimators embedded in a controller structure for a set of critical safety test cases.

- Development and implementation of estimation scheme for mass and inertia parameters. 


\section{Disclosure statement}

No potential conflict of interest was reported by the authors.

\section{Funding}

The European Commission is gratefully acknowledged for their support of the Marie Skłodowska-Curie research and innovation programme ITEAM ${ }^{1}$ (GA 675999). The Research Fund KU Leuven is gratefully acknowledged for its support. The research of F. Naets is funded by a grant from the Research Foundation Flanders (FWO). This research was partially supported by Flanders Make, the strategic research centre for the manufacturing industry.

\section{References}

[1] Bosch. Bosch Automotive Handbook. 9th ed. Stuttgart, Germany: Robert Bosch GmbH; 2014.

[2] Milliken WF, Milliken DL. Race Car Vehicle Dynamics. Warrendale, PA: SAE International; 1995.

[3] Blundell M, Harty D. The Multibody Systems Approach to Vehicle Dynamics. Amsterdam: Elsevier Butterworth-Heinemann; 2004. Automotive Engineering Series; oCLC: 249677935.

[4] Kistler, measuring systems and sensors [https://www.kistler.com/en/]; 2019. (accessed 2019-01-04).

[5] Alatorre Vazquez AG, Charara A, Victorino A. Sideslip Estimation Algorithm Comparison between Euler Angles and Quaternion Approaches with Black Box Vehicle Model. In: 2018 IEEE 15th International Workshop on Advanced Motion Control (AMC). IEEE; 2018. p. 553-559.

[6] Ryu J, Gerdes J. Integrating inertial sensors with gps for vehicle dynamics control. Journal of Dynamic Systems, Measurement, and Control. 2004;:243-254.

[7] Riehm P, Unrau HJ, Gauterin F, et al. 3D brush model to predict longitudinal tyre characteristics. Vehicle System Dynamics. 2019 Jan;57(1):17-43.

[8] Dugoff H, Fancher PS, Segel L. Tire Performance Characteristics Affecting Vehicle Response to Steering And Braking Control Inputs. Ann Arbor, MI, USA: Highway Safety Research Institute, Institute of Science and Technology, The University of Michigan; 1969.

[9] Canudas de Wit C, Tsiotras P. Dynamic tire friction models for vehicle traction control. In: Proceedings of the 38th IEEE Conference on Decision and Control (Cat. No.99CH36304); Vol. 4; Phoenix, AZ, USA. IEEE; 1999. p. 3746-3751.

[10] Åström KJ, Canudas de Wit C. Revisiting the LuGre friction model. IEEE Control Systems. $2008 \mathrm{Jul} ; 28(6): 101-114$.

[11] Pacejka HB, Besselink IJM. Magic Formula Tyre Model with Transient Properties. Vehicle System Dynamics. 1997 Jan;27(sup001):234-249.

[12] Pacejka HB, Bakker E. THE MAGIC FORMULA TYRE MODEL. Vehicle System Dynamics. 1992 Jan;21(sup001):1-18.

[13] Besselink IJ, Schmeitz AJ, Pacejka HB. An improved Magic Formula/Swift tyre model that can handle inflation pressure changes. Vehicle System Dynamics. 2010 Dec; 48(sup1):337-352.

[14] Pacejka HB. Tyre and Vehicle Dynamics. 3rd ed. Elsevier Butterworth-Heinemann; 2012.

\footnotetext{
${ }^{1}$ Interdisciplinary Training Network in Multi-Actuated Ground Vehicles https://iteam-project.net/
} 
[15] Farrelly J, Wellstead P. Estimation of vehicle lateral velocity. In: Int. Conference on Control Applications. IEEE; 1996. p. 552-557.

[16] Kiencke U, Dai A. Observation of lateral vehicle dynamics. In: Control Eng. Pract.; Vol. 5; 1997. p. 1145-1150.

[17] Doumiati M, Victorino A, Lechner D, et al. Observers for vehicle tyre/road forces estimation: Experimental validation. Vehicle System Dynamics. 2010;48(11):1345-1378.

[18] Boulkroune B, v Aalst S, Rademakers E, et al. Vehcile sideslip angle estimation using nonlinear parameter-varying observers. In: Int. Automatic Control Conference; 2015. p. $108-113$.

[19] Grip H, Imsland L, Johansen T, et al. Nonlinear vehicle side-slip angle estimation with friction adaptation. Automatica. 2008;44(3):611-622.

[20] Best M, Gordon T, Dixon P. An Extended Adaptive Kalman Filter for Realtime State Estimation of Vehicle Handling Dynamics. Vehicle System Dynamics. 2000;34(1):57-75.

[21] van Aalst S, Naets F, Boulkroune B, et al. An Adaptive Vehicle Sideslip Estimator for Reliable Estimation in Low and High Excitation Driving. In: Proceeding of the 2018 IFAC Symposium on Control in Transportation Systems; Savona, Italy; 2018.

[22] Pasterkamp WR, Pacejka HB. Application of Neural Networks in the Estimation of Tire/Road Friction Using the Tire as Sensor. Warrendale, PA: SAE International; 1997. SAE Technical Paper 971122.

[23] Pasterkamp WR, Pacejka HB. Optimal Design of Neural Networks for Estimation of Tyre/Road Friction. Vehicle System Dynamics. 1998 Jan;29(sup1):312-321.

[24] Acosta M, Kanarachos S. Tire Force Estimation and Road Grip Recognition Using Extended Kalman Filter, Neural Networks and Recursive Least Squares. Neural Computing and Applications, Springer. 2017;2017:1-21.

[25] Acosta M, Kanarachos S. Teaching a vehicle to autonomously drift: A data-based approach using Neural Networks. Knowledge-Based Systems. 2018 Aug;153:12-28.

[26] Ray L. Nonlinear state and tire force estimation for advanced vehicle control. Transactions on Control Systems Technology. 1995;3(1):117-124.

[27] Yasui Y, Tanaka W, Muragishi Y, et al. Estimation of Lateral Grip Margin Based on Selfaligning Torque for Vehicle Dynamics Enhancement. Warrendale, PA: SAE International; 2004. SAE Technical Paper 2004-01-1070.

[28] Minaki R, Hori Y. Study on cornering stability control based on pneumatic trail estimation by using dual pitman arm type steer-by-wire on electric vehicle. In: 2010 IEEE Vehicle Power and Propulsion Conference; Sep.; 2010. p. 1-6.

[29] Acosta M, Kanarachos S, Fitzpatrick ME. Robust Virtual Sensing for Vehicle Agile Manoeuvring: A Tyre-model-less Approach. IEEE Transactions on Vehicular Technology. 2017;67(3):1894-1908.

[30] Regolin E, Incremona GP, Ferrara A. Longitudinal Vehicle Dynamics Control via Sliding Modes Generation. In: Ferrara A, editor. Sliding Mode Control of Vehicle Dynamics. IET, London; 2017. p. 33-76.

[31] Regolin E, Alatorre A, Zambelli M, et al. A Sliding Mode Virtual Sensor for Wheel Forces Estimation with Accuracy Enhancement via EKF (submitted). Transactions on Vehicular Technology. 2019 (accepted);

[32] Stéphant J, Charara A, Meizel D. Virtual Sensor: Application to Vehicle Sideslip Angle and Transversal Forces. IEEE Transactions on Industrial Electronics. 2004 Apr;51(2):278289.

[33] Simon D. Optimal state estimation: Kalman, $h_{\text {infty }}$, and nonlinear approaches. Hoboken, N.J: Wiley-Interscience; 2006. OCLC: ocm64084871.

[34] Naets F, van Aalst S, Boulkroune B, et al. Design and Experimental Validation of a Stable Two-Stage Estimator for Automotive Sideslip Angle and Tire Parameters. IEEE Transactions on Vehicular Technology. 2017 Nov;66(11):9727-9742.

[35] Matuško J, Petrović I, Perić N. Neural network based tire/road friction force estimation. In: Eng. Appl. Artif. Intell.; Vol. 21; 2008. p. 442-456.

[36] Melzi S, Sabbioni E. On the vehicle sideslip angle estimation through neural networks: 
Numerical and experimental results. In: Mech. Syst. Signal Process.; Vol. 25; 2011. p. 2005-2019.

[37] Tseng HE, Xu L, Hrovat D. Estimation of land vehicle roll and pitch angles. In: Veh. Syst. Dyn.; Vol. 45; 2007. p. 433-443.

[38] Guo H, Chen H, Song T. Tire-road forces estimation based on sliding mode observer. In: Proceedings of the 2009 IEEE International Conference on Mechatronics and Automation; Vol. 08; 2009.

[39] Baffet G, Charara A, Lechner D. Experimental evaluation of a sliding mode observer for tire-road forces and an extended kalman filter for vehicle sideslip angle. In: Proceedings of the 46th IEEE Conference on Decision and Control; Vol. 12; 2007.

[40] Segel M. Theoretical Prediction and Experimental Substantiation of the Response of the Automobile to Steering Control. In: Proceedings of the Inst. of Mechanical Engineers: Automobile Division; Vol. 10; Jan.; 1956. p. 310-330.

[41] Albinsson A, Bruzelius F, Jonasson M, et al. Tire Force Estimation Utilizing Wheel Torque Measurements and Validation in Simulations and Experiments. In: 12th International Symposium on Advanced Control; Sep.; 2014. p. 294-299.

[42] Hashemi E, Zarringhalam R, Khajepour A, et al. Real-time estimation of the road bank and grade angles with unknown input observers. Vehicle System Dynamics. 2017 May; $55(5): 648-667$.

[43] Martin PG, Hall GW, Crandall JR, et al. Measuring the Acceleration of a Rigid Body. Shock and Vibration. 1998;5(4):211-224.

[44] Doumiati M, Charara A, Victorino A, et al. Vehicle dynamics estimation using Kalman filtering: Experimental validation. London Hoboken, N.J: ISTE; 2013. Automation - Control and Industrial Engineering Series; oCLC: 829902596.

[45] Smith G. The Development of an Efficient Tyre Testing Procedure to Gather Data for the Parameterisation of Magic Formula 6.1 Tyre Models, PhD Thesis. Coventry University; 2018.

[46] Dufournier. Skid Trailer: For Tire Characterization and Labelling. Dufournier Technologies; 2012.

[47] Schmeitz A. TNO's MF-Tyre / MF-Swift and the Delft-Tyre Toolchain. Simpack News March 2014. Simpack; 2014.

[48] Heißing B, Ersoy M, editors. Chassis handbook: Fundamentals, driving dynamics, components, mechatronics, perspectives. 1st ed. Wiesbaden: Vieweg + Teubner; 2011. ATZ; oCLC: ocn832319879.

[49] Bartolini G, Ferrara A, Usai E. Chattering Avoidance by Second-Order Sliding Mode Control. IEEE Trans Automat Control. 1998 Feb;43(2):241-246.

[50] Regolin E, Zambelli M, Ferrara A. Wheel Forces Estimation via Adaptive Sub-Optimal Second Order Sliding Mode Observers. In: 2017 XXVI International Conference on Information, Communication and Automation Technologies (ICAT); Oct.; Sarajevo, Bosnia and Herzegovina; 2017. p. 1-6.

[51] Acosta M, Gladstone M, Prins J, et al. On Tyre Force Virtual Sensing for Future Automated Vehicle-Based Objective Tyre Testing (AVBOTT). Vehicle System Dynamics. 2018 Nov;:27.

[52] Selmanaj D, Corno M, Panzani G, et al. Vehicle sideslip estimation: A kinematic based approach. Control Engineering Practice. 2017 Oct;67:1-12.

[53] Madhusudhanan AK, Corno M, Holweg E. Vehicle sideslip estimator using load sensing bearings. Control Engineering Practice. 2016 Sep;54:46-57. 